\title{
Temperature-driven narrowing of the insulating gap as a precursor of the insulator-to-metal transition: Implications for the electronic structure of solids
}

\author{
Vassiliy Lubchenko ${ }^{1,2, *}$ and Arkady Kurnosov ${ }^{1}$ \\ ${ }^{1}$ Department of Chemistry, University of Houston, Houston, TX 77204-5003 \\ ${ }^{2}$ Department of Physics, University of Houston, Houston, TX r7204-5005
}

(Dated: October 1, 2018)

\begin{abstract}
We present a microscopic picture rationalizing the surprisingly steep decrease of the band gap with temperature in insulators, crystalline or otherwise. The gap narrowing largely results from fluctuations of long-wavelength optical phonons - when the latter are present - or their disordered analogs, if the material is amorphous. We elaborate on this notion to show that possibly with the exception of weakly bound solids made of closed-shell atoms, the existence of an insulating gap or pseudo-gap in a periodic solid implies that optical phonons must be present, too. This means that in an insulating solid, the primitive cell must have at least two atoms and/or that a charge density wave is present. As a corollary, a (periodic) elemental solid whose primitive unit contains only one atom will ordinarily be a metal, possibly unless the element belongs to group 18 of the periodic table, consistent with observation. Some implications of the present results for quantum solids are briefly discussed, such as that the ground state of the Wigner crystal must be anti-ferromagnetic. A simple field theory of the metal-insulator transition is constructed that ties long-wavelength optical vibrations with fluctuations of an order parameter for the metal-insulator transition; symmetrybreaking aspects of the latter transition are thereby highlighted.
\end{abstract}

\section{MOTIVATION}

The temperature-dependence of the insulating gap, which is exemplified by the data for a small number of crystalline and amorphous compounds in Fig. 1 is significant for quantitative descriptions of the electrical conductivity and many other electronic phenomena. In a substantial temperature interval, the dependence can be described by a simple linear law:

$$
E_{g} \approx E_{g, 0}-\gamma T
$$

where $E_{g, 0}$ and $\gamma$ are numerical constants. The absorption edge is not sharp even in crystalline compounds because optical transitions are much faster than atomic motions; the instantaneous positions of the ions are disordered already owing to thermal motions, let along when the solid is amorphous. The so smeared optical edge is often called the Urbach $\underline{1} \underline{\underline{2}}$ tail (or edge), or Lifshitz tail $\underline{\underline{3}}$

Because of the edge smearing, one customarily defines the optical gap at a given value of the absorption coefficient $\alpha$, the smaller the $\alpha$ the greater the coefficient $\gamma$ from Eq. (1). This is illustrated in Fig. 2 the original data can be found in Refs 1, 2, 4, and 7. We show the values of the coefficient $\gamma$ that are pertinent to activated charge transport; these correspond to optical transitions between "borderline" states that separate delocalized from localized states in the respective bands. This will be discussed in more detail in the bulk of the paper.

The pertinent values of $\gamma$ are surprisingly large, as in Fig. 10 They imply that temperature changes on the order of a few hundredths of $\mathrm{eV}$ cause changes in the electronic structure that are an order of magnitude larger. In addition to raising mechanistic questions, this strong effect has direct consequences for estimating the (temperature dependent) electrical conductivity $\sigma(T)$ in the

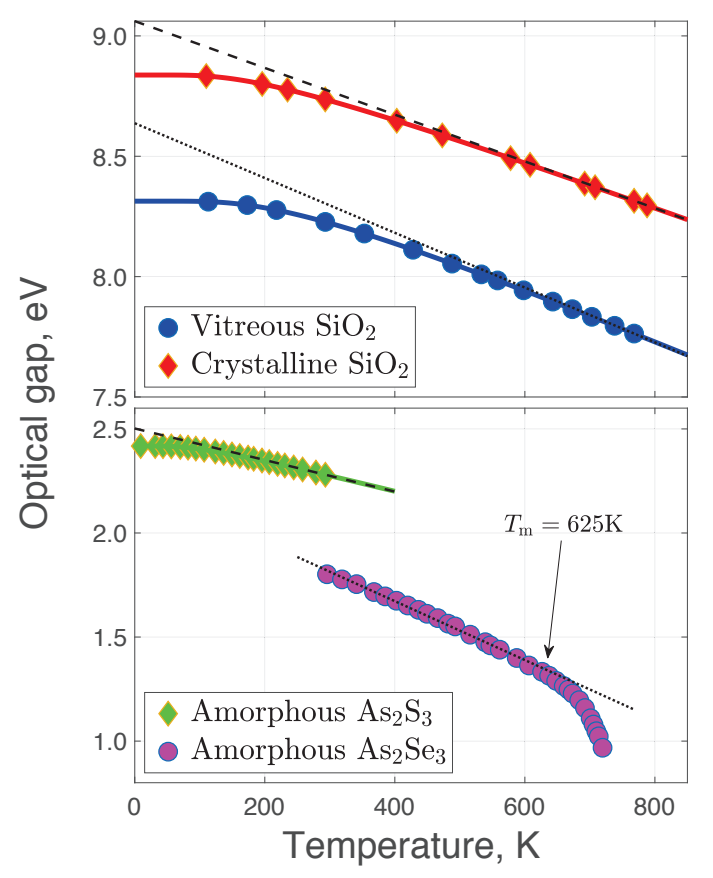

FIG. 1. Optical gaps in vitreous and crystalline $\mathrm{SiO}_{2}$ (top), and amorphous $\mathrm{As}_{2} \mathrm{~S}_{3}$ and $\mathrm{As}_{2} \mathrm{Se}_{3}$ (bottom) as functions of temperature. The quartz data were inferred from Ref. 4, $\mathrm{As}_{2} \mathrm{~S}_{3}$ from Ref. 5 and amorphous $\mathrm{As}_{2} \mathrm{Se}_{3}$ from Ref. 6. The choice of the respective absorption coefficients, listed in Table [1 is explained in Section II] The solid lines represent fits obtained using the present description, see Section III The corresponding parameters are also listed in Table [1 The straight lines correspond with the high- $T$ behavior from Eq. (1).

thermally activated regime. Because the effective activation energy for charge transport could be as large as 


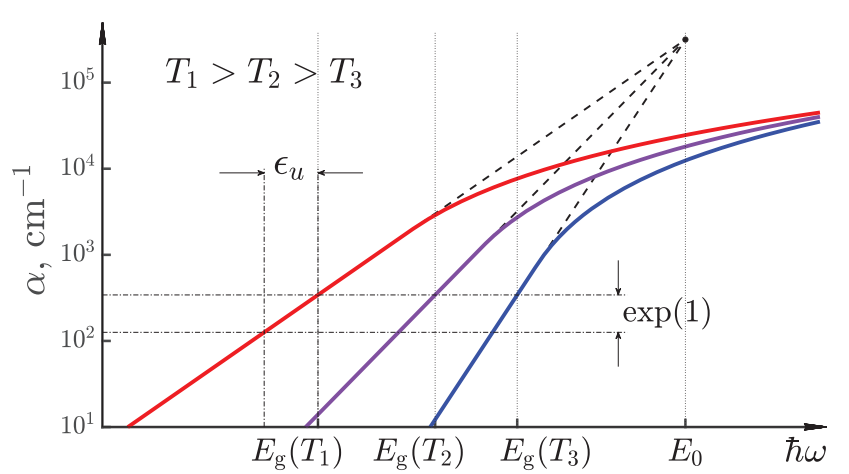

FIG. 2. Typical behavior of the optical absorption spectrum as a function of temperature. $\alpha$ is the standard absorption coefficient. The width of the exponential tail of localized states is seen to change synchronously with the mobility edge.

$E_{g} / 2$, the $(-\gamma T)$ term in Eq. (11) could lead to an effective increase of the prefactor in the activation rate by as much as $e^{\gamma / 2}$, the latter quantities listed in Table I. The actual increase is usually smaller because only one of the carriers dominates; still it is usually on the order of $10^{2}$, as will be discussed in a separate submission. Ignoring such a large factor could throw off one's estimates of the density of states of charge carriers and/or their mobility by an amount that would be difficult to write off on one's being agnostic as to the detailed shape of the electronic density of states or the precise strength of electron-lattice coupling.

Many substances exhibit a remarkable behavior, exemplified in Fig. 2. where the temperature-driven narrowing of the band and the broadening of the exponential tail of localized states occur in a synchronous fashion. More specifically, the onset energy of the tail of the localized states is exactly proportional to the width of the tail $\underline{8}$ This remarkable behavior may or may not be fully universal; at least in some cases deviations can be accounted for by the difficulty in isolating the amount of midgap absorption due to impurities $\underline{\underline{9}}$

Existing views on the microscopic origin of the temperature-driven narrowing of the band gap seem to hark back to the work of Fan $\underline{10}, 11$ dating to the early 1950s. For polar substances, Fan co-opts Fröhlich's formalism 12,13 for the electron-lattice coupling in ionic substances to conclude that in the presence of an electron, the conduction band would be stabilized owing to fluctuations of the lattice's polarization, much like that leading to the attractive van der Waals force. In a rather distinct treatment of non-polar substances, the latter author assumes that optical phonons, if any, are not even important and subsequently estimates the gap narrowing exclusively due to interaction between electrons and acoustic phonons. Hereby, the issue of the surprisingly large value of $\gamma$ is thus relegated to the detailed question of electron scattering off lattice vibrations.

Early efforts to explain the Urbach tail also emphasized the importance of lattice polarization, such as that

\begin{tabular}{|l|c|c|c|c|c|}
\hline & $\gamma, \mathrm{eV} / \mathrm{K}$ & $e^{\gamma / 2}$ & $\alpha, \mathrm{cm}^{-1}$ & $\omega, \mathrm{K}$ & $V, \mathrm{eV}$ \\
\hline$a-\mathrm{SiO}_{2}$ & $1.15 \cdot 10^{-3}$ & 785 & $2.40 \cdot 10^{2}$ & 674 & 11.6 \\
\hline$c-\mathrm{SiO}_{2}$ & $0.98 \cdot 10^{-3}$ & 303 & $4.70 \cdot 10^{3}$ & 535 & 10.7 \\
\hline$a-\mathrm{As}_{2} \mathrm{~S}_{3}$ & $0.78 \cdot 10^{-3}$ & 93 & $2.35 \cdot 10^{2}$ & 275 & 4.8 \\
\hline$a-\mathrm{As}_{2} \mathrm{Se}_{3}$ & $1.41 \cdot 10^{-3}$ & 3735 & N/A & N/A & 6.3 \\
\hline
\end{tabular}

TABLE I. The quantity $\gamma$ is from Eq. (1). The quantity $e^{\gamma / 2}$ is computed using the dimensionless $\gamma$. The quantity $\alpha$ gives the value of the absorption coefficient, at which the optical gap was determined, see Eq. (15). Values $T_{\mathrm{m}}$ used for the purpose of fitting are, respectively, $1986 \mathrm{~K}$ for $\mathrm{SiO}_{2}{ }^{15}, 585 \mathrm{~K}$ for $\mathrm{As}_{2} \mathrm{~S}_{3}{ }^{9,15}$ and $625 \mathrm{~K}$ for $\mathrm{As}_{2} \mathrm{Se}_{3}$.

arising from excitonic effects. Subsequent studies however seem to shift their focus to the physics of Anderson localization, by looking at the electronic motion subject to a random potential. Multiple studies spanning decades indicate that the "simple" exponential shape of the optical edge is nothing but: Within a general, even if phenomenological picture, the exponential tail can be viewed as an intermediate regime between weak and very strong localization of electrons subject to a random potential. To account for the substantial spectral range occupied by the Urbach tail, one must however assume that the spatial correlations between the fluctuations of the random potential must be strong within a small multiple of the atomic size but decay rapidly beyond that, more rapidly than, for instance, exponentially. The microscopic mechanism behind this puzzling observation is not clear. Still, relatively explicit description modeling polaronic effects indicate that the exponential density of localized states is in fact robust, subject to modest non-adiabatic effects $\frac{14}{1}$

Here we present a qualitative microscopic picture that directly connects the phenomena of both band narrowing and the tail of localized states with the atomic motions. In turn, these are tied to the fluctuations of the optical phonons. The effective random potential is seen as stemming directly from the disorder in the bond length. The coupling with vibrations comes about because both the electronic transfer integral and the dipole moment for electronic motions depends on the bond length.

We elucidate the apparent, seemingly intrinsic connection between the presence of an insulating gap, on the one hand, and the existence of optical phonons, on the other hand. The physical reason behind this connection is relatively lucid: In the presence of bonding that is not closed-shell, electronic excitations across the forbidden gap and polarization must both involve charge transfer between atoms. Accordingly, the existence of an insulating gap or pseudo-gap necessarily implies that there are at least two orbitals centered on distinct atoms within the primitive cell and so the optical phonons must be present, too. A corollary of this observation is that if the optical phonons are absent, the solid is metallic. Hence an elemental solid that has one atom per primitive cell will be ordinarily a metal. An arguable exception to this rule would be when such an elemental solid hosts a charge density wave; still such a solid, strictly speak- 
ing, should be regarded as hosting at least two inequivalent atoms per primitive cell and would, in fact, possess optical phonons. These notions are consistent with experience. Applying the present argument to the Wigner crystal leads one to conclude that the ground state of this quantum solid must be anti-ferromagnetic, consistent with quantum Monte Carlo simulations ${ }^{16.17}$

As an additional dividend, the present picture allows one to build a field theory, for a substantial class of materials, to show that the optical phonons can be thought of as fluctuations of the order parameter for the metalinsulator transition. The order parameter must be at least two-dimensional, the two components reflecting, respectively, the strength of spatial inhomogeneity of the charge distribution on the atomic sites and interatomic spacings. (The inhomogeneity can be thought of spatially-inhomogeneous bond saturation.) As such, the gap narrowing can be viewed rather generally as signaling the eventual transition to a metallic state. In the present treatment, the metal-to-insulator transition is naturally a symmetry breaking transition and could be either continuous or discontinuous. Despite the order parameter being vectorial and possibly exhibiting a continuous symmetry in the symmetric state, the symmetry breaking can be explicitly shown to not give rise to Goldstone modes. Instead, the optical phonons, which emerge following the symmetry breaking are gapped excitations as they should be.

\section{THE $T$-DEPENDENCE OF THE OPTICAL GAP AND THE GAP EDGE, AND THE IDENTITY OF THE CHARGE TRANSFER STATES}

The main purpose of this Section is to substantiate our earlier statement is that the $T$-dependence of the band gap is surprisingly strong, with implications for calculations of the electrical conductivity. The dependence of the optical gap can be described by a simple phenomenological relation: $: 7,10,11,18$

$$
E_{g}=C_{1}-\left(\gamma / T_{0}\right) \operatorname{coth}\left(T_{0} / T\right)
$$

where $C_{1}$ and $T_{0}$ are numerical constants. At sufficiently high temperatures, this implies a simpler yet, linear $T$ dependence in Eq. (11). Functional forms other than that in Eq. (2) often work, too $\stackrel{19,20}{=}$ As mentioned in Section 【. one customarily determines the optical gap at a fixed value of the absorption coefficient $\alpha$ because the optical edge is not sharp. The coefficients $C_{1}$ and $C_{2}$ thus depend on the precise value of $\alpha$.

In a relatively broad energy range - often corresponding to several orders of magnitude in terms of $\alpha$ - the optical edge apparently exhibits an approximately exponential shape:

$$
\alpha(E)=\alpha_{0} e^{\left(E-E_{0}\right) / \epsilon_{U}}
$$

where the quantity $\epsilon_{U}$ reflects the width of absorption edge. The temperature dependence of the width of the Urbach tail is often well described by a phenomenological relation:

$$
\epsilon_{U}=C \omega \operatorname{coth}(\omega / T)
$$

where the constant $C$ is numerically close to two $\underline{\underline{4}}$

Whether or not the substance obeys the noteworthy trend illustrated in Fig. 2, it is clear from Eqs. (2), (3) and (4) that the value of $\gamma$ generally depends on the value of the absorption coefficient at which the optical gap is defined. One may further ask: What is the value of the band gap that is relevant in the context of electrical conduction or whether such a question is well-posed in the first place.

To avoid ambiguity we note that, strictly speaking, the exponential shape of the absorption edge is not equivalent to the edges of the bands themselves being exponential. Still, photo-conductivity experiments by Monroe and Kastner $\underline{\underline{ }}$ strongly suggest that the individual bands do have several decades worth of density of states in the form of exponential tails. It is straightforward to show that the width of the exponential tail in the optical absorption is equal to the sum of the widths $\epsilon_{c}$ and $\epsilon_{v}$ of the exponential edges of the conduction and valence bands, respectively:

$$
\epsilon_{U}=\epsilon_{c}+\epsilon_{v} .
$$

We now briefly discuss two specific views on charge transport in semiconductors $\stackrel{22,23}{=}$ In one model, the conduction is solely due to delocalized carriers. Hence the energy scale $E_{\sigma}$ in the activated part of the electrical conductivity, $e^{-E_{\sigma} / k_{B} T}$, is determined by the energy difference $\left(E_{c}-\mu\right)$ between the bottom of the conduction (mobility) band and the chemical potential $\mu$, for the electrons, and the energy difference $\left(\mu-E_{v}\right)$ between the chemical potential and the ceiling of the valence (mobility) band, for the holes.

In an alternative picture, charge transport is carried out via activated hopping of small polarons. These quasiparticles result from self-trapping of electrons, $\stackrel{24}{\underline{w}}$ which requires sufficiently strong electron-phonon coupling and would be enhanced by the presence of disorder $\stackrel{25,26}{=} \mathrm{It}$ is deemed that the materials of the type exemplified in Fig. 1 do in fact conduct electricity primarily with small polarons, but there does not seem to be complete agreement on this issue, see Refs. 22, 23, and 27 for a detailed discussion.

In the most basic version of the small polaron picture, the activation part of the $T$-dependence of the electrical conductivity due to, say, the electron polarons is determined by energy cost of putting an electron in the small polaron band, $\left(E_{e, \text { pol }}-\mu\right)$, and the activation energy needed to reach the pertinent mobility band from the polaron state: $\left(E_{c}-E_{e, \mathrm{pol}}\right)$. As in the delocalizedcarrier picture, the activation energy is $\left(E_{c}-\mu\right)$. In the presence of a "smeared" edge in the density of states, this 
relatively simple picture deserves some additional discussion. Indeed, the activation exponent for transport must be averaged with respect to the DOS of the localized states that could be transition states for polaron hopping. Considering electronic polarons for concreteness, one may write

$$
\left\langle\nu e^{-\left(E-E_{e, \mathrm{pol}}\right) / k_{B} T}\right\rangle \propto \int d E d^{3} \boldsymbol{r} \nu e^{-E / k_{B} T} n(E, \boldsymbol{r}),
$$

where the coordinate dependence in the density of states $n(E, \boldsymbol{r})$ reflects that the wave-function overlap between the initial and transition states will generally depend on spatial separation between the two. The specifics of averaging depend on the detailed nature of the disorder. Two limiting cases are relatively straightforward to describe:

(1) If the disorder is caused exclusively by vibrations of the lattice, then the effective density of states $n(E, \boldsymbol{r})$ becomes coordinate-independent. This is because the waiting time for polaron hopping is much longer than the time scale of the lattice vibrations. Thus every configuration of the lattice will be sampled locally so that the volume-integrated density of states is proportional to the bulk density of states $e^{E / \epsilon_{c}}$ within the tail:

$$
\int d^{3} \boldsymbol{r} n_{\text {dynamic }}(E, \boldsymbol{r}) \propto e^{\left(E-E_{c}\right) / \epsilon_{c}}
$$

where $E<E_{c}$. Note that the prefactor $\nu$ for the rate of hopping is largely determined by the vibrational frequency: The electronic wave function overlap is large implying the hopping is adiabatic.

Now, according to the discussion of Eq. (3), $\epsilon_{c}$ is only slightly greater, if at all, than the temperature. This implies that although the integral in Eq. (6) is dominated by the states near $E_{c}$, a broad spectral range of states will generally contribute to transport as transition states for polaron hopping. In any event, insofar as $\epsilon_{c}<k_{B} T$, the overall activation energy scale for the polaron hopping is still determined by the position of the pertinent mobility band since the integrand in Eq. (77) peaks out near $E_{c}$ : The density of states in the mobility band, i.e. at $E>E_{c}$, depends on energy much more slowly than the Boltzmann weight $e^{-E / k_{B} T}$.

(2) When the disorder is exclusively static, the coordinate-averaged effective density of transition states decreases away from the band edge, with decreasing energy, than the exponential density of states in the r.h.s. of Eq. (7). This is because a tail state at a given energy is no longer guaranteed to be near the initial location of the polaron and so the probability rapidly decreases with the depth $\left(E_{c}-E\right)$ of the state in question. Indeed, the electronic wave-function overlap between the initial and transition states will go (for electrons) as $e^{-r / l}$, where $l$ is the localization length of the orbital representing the transition state. Thus the spatial averaging in Eq. (6) yields a factor $e^{-\bar{r} / l}$, where the typical distance $\bar{r}$ to the nearest transition state at energy $E$ is determined from the bulk density of states from the r.h.s. of Eq. (7) ac- cording to $1 / \bar{r}^{3} \sim l^{-3} e^{\left(E-E_{c}\right) / \epsilon_{c}}$. This yields

$$
\int d^{3} \boldsymbol{r} n_{\text {static }}(E, \boldsymbol{r}) \propto \exp \left[-e^{\left(E_{c}-E\right) / 3 \epsilon_{c}}\right],
$$

where $E<E_{c}$, as before. The r.h.s. is only a (rough) upper bound, and increasingly so for smaller values of $E$. This is because for larger values of $r$, and hence large values of $\left(E_{c}-E\right)$, the transition will be increasingly more non-adiabatic as the transition state wave-function becomes more and more localized. Consequently, the frequency prefactor for the hopping rate $\nu$ will be decreased by the corresponding Franck-Condon factor for the lattice vibrations. Thus the dependence of the coordinateintegrated density of transition states on $E$ on energy is even stronger than that implied by the r.h.s. of Eq. (8). Because of this very strong dependence, the lower limit for the energy integration in Eq. (6) is now effectively pinned at or just below the mobility edge. The coordinate-averaged density of states for the transition states for polaron hopping in amorphous materials will interpolate between Eqs. (7) and (8), the detailed answer depending on the interplay of the static and dynamic disorder.

In any event, we conclude that the activation energy for the electrical conductivity is determined by the locations of the mobility edges $E_{c}$ and $E_{v}$. Consequently, one may peg the relevant value of the absorption strength at the corresponding energy difference:

$$
E_{g}=E_{c}-E_{v}
$$

The spacing between the mobility edges is conventionally determined in absorption experiments by first fitting the large- $\alpha$ portions of the absorption spectra by a quadratic functional form $\left(E-\omega_{b}\right)^{2}$ where $\omega_{b}$ is a fitting parameter. Absorption strength at $\omega_{b}$ is often numerically close to $10^{3} \mathrm{~cm}^{-1}$. The aforementioned quadratic form is expected 28,29 when the conduction and valence bands, respectively, behave like those in a periodic 3D solid: $\propto\left(E-E_{c}\right)^{1 / 2}$ and $\propto\left(E-E_{v}\right)^{1 / 2}$. We however believe that this empirical procedure overestimates the band gap because the states in the mobility bands are stabilized owing to electron-lattice interactions as discussed in Section [I the amount of stabilization increasing toward the band edge. (This immediately follows from the expression for the second-order term in the perturbation theory ${ }^{30}$ ) This will lead to deviations from the simple square-root shape of the band edge expected of a strictly periodic solid in which nuclei do not vibrate altogether.

Here, instead, we determine the spacing between the mobility edges by utilizing the results of S. John et al $\stackrel{31}{33}$ obtained for a one-electron model, in which the electron is subject to a random potential $V(x)$ :

$$
\left[-\hbar^{2} / 2 m+V(x)\right] \psi(x)=E \psi(x) .
$$

By construction, $\langle V(x)\rangle=0$, while the corresponding auto-correlation function decays in a Gaussian fashion 
with distance:

$$
\langle V(x) V(0)\rangle=V_{\mathrm{rms}}^{2} e^{-x^{2} / L^{2}} .
$$

The shape of the tail of the localized shape depends on the detailed functional form of the auto-correlation. A substantial exponential tail is predicted for functional forms that decay faster than the Gaussian, but not slower. With this proviso, the model yields both the stabilization $\Delta E$ of the band edges and the width $\epsilon$ of the exponential tail, the two being exactly proportional to each other with a universal coefficient that depends on the dimensionality of space, very much consistent with the behavior seen in Fig. 2. According to Eqs. (A4) of Ref. 32,

$$
\Delta E=-V_{\mathrm{rms}}^{2} / 2 \epsilon_{L},
$$

while Eq. (6) of Ref. 31 states that

$$
\epsilon=V_{\mathrm{rms}}^{2} / 14.4 \epsilon_{L} .
$$

Here the quantity $\epsilon_{L}$ reflects the cost of localizing the particle within a region of size $L$ :

$$
\epsilon_{L} \equiv \hbar^{2} / 2 m L^{2} \text {. }
$$

In conjunction with Eq. (3), this immediately yields a simple relation:

$$
\epsilon_{U}=\left(E_{0}-E_{g}\right) / 7.2 .
$$

Notwithstanding the approximate nature of the calculation in Refs. 31 33, the quantities $\epsilon_{U}$ and $\left(E_{0}-E_{g}\right)$ are obtained internally-consistently using a constructive argument. Thus the above relation between the two quantities is expected to be less sensitive to the approximations made in Refs. 31 33 than the individual quantities themselves. In any event - and consistent with expectationthe so determined gap is lower than than obtained using the purely phenomenological protocol described above, i.e., at values of $\alpha$ close $10^{2} \mathrm{~cm}^{-1}$. This yields rather substantial values for the coefficient $\gamma$ from Eq. (1). As discussed in Section [1] this has significant consequences for quantitative estimates of electrical conductivity in semiconductors.

\section{BAND NARROWING AS A RESULT OF FLUCTUATIONS OF OPTICAL VIBRATIONS: A QUALITATIVE TREATMENT}

Next we outline a simple structural model for the coupling of the band gap to lattice distortion. Our main focus is on two relatively distinct types on insulators: (1) Non-polar or weakly polar semiconductors exhibiting spatial inhomogeneous bond saturation, such as the chalcogenide compounds $\mathrm{As}_{2} \mathrm{Se}_{3}$ and $\mathrm{As}_{2} \mathrm{~S}_{3}$, see Fig. 1. In such compounds, there are two types of interactions: covalent bonds and the secondary interactions 34 that are stronger than Van der Walls but, technically, are closedshell. The total number of each is roughly the same 35 (2) Ionic insulators such as oxides or halides, such as $\mathrm{SiO}_{2}$, see Fig. 1] We will also briefly touch on yet another class of compounds. These are non-polar or weakly polar semiconductors that exhibit largely uniform bond strength throughout, such as $\mathrm{Si}, \mathrm{Ge}$, or GaAs. All individual bonds in the latter compounds are well described as canonical covalent bonds of order 1 .

Type 1 compounds are well represented by elemental arsenic and various chalcogenide alloys: These can be thought of approximately as $p p \sigma$-bonded networks exhibiting distorted octahedral coordination $\stackrel{35}{ }$ In turn, these distorted structures can be thought of as symmetry lowered versions of parent structures defined on the simple cubic lattice, possibly with vacancies. In the absence of $s p$-mixing, one can think of the individual chains comprising the cubic lattice as Peierls unstable toward dimerization $\sqrt[35-37]{ }$ The presence of $s p$-mixing does affect the eventual geometry of the distorted lattice but does not change the picture qualitatively. This is consistent with the $s$ - and $p$-bands being well separated in the electronic density of states for these compounds, the $s$-band being completely filled, see for instance Ref. 38 .

Qualitatively, the electronic transfer integral scales exponentially with the interatomic distance

$$
t_{\mathrm{e}} \approx t_{0} e^{-A r},
$$

for an interatomic distance $r$ that is not too small and $A$ is on the order of the inverse Bohr radius; the quantity $A r$ exceeds one but is certainly less than 10. The value of $t$ is of the order $\mathrm{eV}$. To give a specific example, $A \approx 1.2 \AA^{-1}$ for the As-Se bond, as can be inferred 39 from the semi-empirical package MOPAC $\stackrel{40,41}{ }$ In the amorphous $\mathrm{As}_{2} \mathrm{Se}_{3}$ compound, the stronger and weaker bond are typically of length $r_{1}=2.4$ Åand $r_{2}=3.6 \AA$, respectively $\stackrel{38}{\underline{3}}$ Direct sampling of the amorphous $\mathrm{As}_{2} \mathrm{Se}_{3}$ samples generated in Ref. $\underline{42}$ indicates the corresponding $p p \sigma$ transfer integrals are typically $6 \mathrm{eV}$ and 1-2 eV for the strong and weak As-Se bond, respectively.

Clearly, fluctuations in the inter-atomic spacings, due lattice vibrations, will modulate the magnitude of the transfer matrix elements from Eq. (16). In the BornOppenheimer approximation, electrons will be subject to a static random potential such as that in Eq. (10). To find this connection, we consider for concreteness a $p p \sigma$-bonded solid made of $1 \mathrm{D}$ chains at half-filling. Each atom houses three $p$ orbitals; the chains along the $x$ axis are made of $p_{x}$ orbitals etc. It will suffice for now to assume that there is no $s p$-mixing, so that the chains are non-interacting. For each chain, we write down a Hückel energy function, one orbital per site:

$$
\begin{gathered}
\mathcal{H}=\sum_{n} \sum_{s= \pm 1 / 2}\left[-t_{n, n+1}\left(c_{n, s}^{\dagger} c_{n+1, s}+c_{n+1, s}^{\dagger} c_{n, s}\right)\right. \\
\left.+\varepsilon_{n} c_{n, s}^{\dagger} c_{n, s}\right],
\end{gathered}
$$

where $c_{n, s}^{\dagger}\left(c_{n, s}\right)$ creates (annihilates) an electron on site $n$ with spin $s$. We will assume that the electrons are 
non-interacting. Within limits, such interactions can be thought of as renormalizing the transfer integrals $t_{n, n+1}$ and the on-site energies $\varepsilon_{n} \cdot 35$ The transfer integrals are assumed to scale with the interatomic spacing as in Eq. (16).

The chain described by Eq. (17), at half-filling, is unstable toward Peierls dimerization,,-45 if the electronegativity variation, as embodied in the on-site energies $\varepsilon_{n}$,

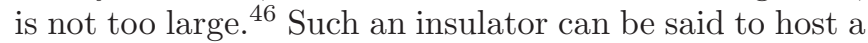
"bond-order" charge density wave. The dimerized chain contains two atoms per repeat unit and, also, supports optical phonons. It is convenient to introduce a specific modulation pattern for the electronegativity, which has the same periodicity as the dimerized chain:

$$
\varepsilon_{n}=(-1)^{n} \varepsilon \text {. }
$$

If desired, one may create a primitive unit that spans more than two atoms, by employing a different value for the electron filling and the corresponding period of the electronegativity variation.

We reiterate that for a sufficiently small $\varepsilon$, we have an insulator of type 1 . Otherwise, i.e., when $|\varepsilon| \gtrsim \bar{t}, \underline{46}$ the chain exemplifies an insulator of type 2 . In a dimerized chain, the hopping matrix element alternates between two values:

$$
t_{n, n+1} \equiv \bar{t}+(-1)^{n} t, \quad|t| \leq \bar{t}
$$

In the existing notation, $t=\left(t_{1}-t_{2}\right) / 2$ and $\bar{t}=$ $\left(t_{1}+t_{2}\right) / 2$. Hamiltonian (17) can be broken up, in a standard fashion, into a set of $N / 2$ non-interacting twostate systems. Each such system operates on two plane waves, each at wavevector $k$, spanning the odd- and evennumbered sites, respectively: $\underline{45}$

$$
\begin{aligned}
\left|\psi_{k}^{\text {odd }}\right\rangle & =(2 / N)^{1 / 2} \sum_{n}\left|\psi_{2 n+1}\right\rangle e^{i k a(2 n+1)} \\
\left|\psi_{k}^{\text {even }}\right\rangle & =(2 / N)^{1 / 2} \sum_{n}\left|\psi_{2 n}\right\rangle e^{i k a 2 n} .
\end{aligned}
$$

The kets $\left|\psi_{n}\right\rangle$ stand for the basis set of the matrix $H_{m n}$ defining the tight-binding Hamiltonian from Eq. (17): $\mathcal{H} \equiv \sum_{m n, s} H_{m n} c_{m, s}^{\dagger} c_{n, s}$. The two-level system, for each value of $k$, is defined by the matrix:

$$
\left.\begin{array}{c|c}
-\varepsilon & -2 \bar{t} \cos k a+2 i t \sin k a \\
\hline-2 \bar{t} \cos k a-2 i t \sin k a & \varepsilon
\end{array}\right),
$$

where $a$ is the lattice spacing. The resulting spectrum is:

$$
E_{k}=\mp\left[\varepsilon^{2}+4 t^{2}+4\left(\bar{t}^{2}-t^{2}\right) \cos ^{2} k a\right]^{1 / 2},
$$

see the sketch in Fig. 3. The spectrum is doubly degenerate: $E_{k}=E_{-k}$ and possesses a band gap:

$$
E_{g}=2 \sqrt{\left(t_{1}-t_{2}\right)^{2}+\epsilon^{2}} .
$$

The Hamiltonian (17), which is defined for fixed values of $\bar{t}, t$, and $\varepsilon$, can be generalized for parameters $t(x)$ and

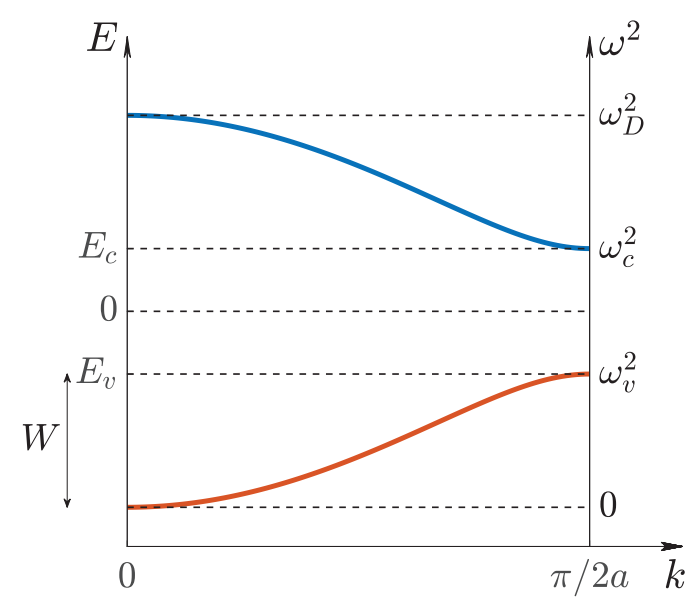

FIG. 3. The electronic and vibrational spectrum of a dimerized chain. The dimerization could be due to an alternation in the strength and/or identity of the ion.

$\epsilon(x)$ slowly varying with the spatial coordinate $x$ along the chain ${ }^{46-48}$ This is conveniently done by shifting the wavevector reference in the two-level Hamiltonian (22) to the edge of the Brillouin zone of the original Hamiltonian (17), $(k-\pi / 2 a) \rightarrow k$, and then expanding around $k=0$ :

$$
\mathcal{H}=-i 2 \bar{t} a \hat{\sigma}_{3} \frac{\partial}{\partial x}-2 t(x) \hat{\sigma}_{1}-\varepsilon(x) \hat{\sigma}_{2}
$$

where the operators $\hat{\sigma}_{i}$ are the standard Pauli matrices. We have relabeled the latter, $\left(\sigma_{1}, \sigma_{2}, \sigma_{3}\right) \rightarrow\left(\sigma_{2}, \sigma_{3}, \sigma_{1}\right)$, so that Hamiltonian (25) now operates on spinors with components $\psi_{1}=\left(\psi^{\text {odd }}+\psi^{\text {even }}\right) / \sqrt{2}$ and $\psi_{1}=\left(\psi^{\text {odd }}-\right.$ $\left.\psi^{\text {even }}\right) / \sqrt{2}$. These two correspond with the filled and empty orbitals of the non-dimerized chain and sometimes are called the right and left movers. This is because of the aforementioned degeneracy $E_{k}=E_{-k}$, and the equivalence of points $k$ and $k+\pi / a$.

It is convenient to rotate the basis set in Eq. (25) in the $\left(\sigma_{1}, \sigma_{2}\right)$ plane so that the corresponding part of the Hamiltonian is directed along, say, direction $\sigma_{1}$. Keeping in mind that the rotation angle $\varphi$ is $x$-dependent, one obtains:

$$
\mathcal{H}=-i 2 \bar{t} a \hat{\sigma}_{3} \frac{\partial}{\partial x}-\Delta \hat{\sigma}_{1}-\bar{t} a \frac{\partial \varphi}{\partial x}
$$

where

$$
\Delta \equiv\left[(2 t)^{2}+\varepsilon^{2}\right]^{1 / 2}
$$

and

$$
\frac{\partial \varphi}{\partial x}=\frac{2}{\Delta^{2}}\left(\varepsilon \frac{\partial t}{\partial x}-t \frac{\partial \varepsilon}{\partial x}\right)
$$

The Schrödinger equation for the Hamiltonian (26), which operates on the spinor with components $\psi_{1}$ and 
$\psi_{2}$, can be profitably recast in terms of the linear combinations $\psi_{ \pm}=\left(\psi_{1} \pm i \psi_{2}\right) / \sqrt{2}$ :

$$
\begin{aligned}
i\left(-2 \bar{t} a \frac{\partial}{\partial x}+\Delta\right) \psi_{-} & =\left(E+\bar{t} a \frac{\partial \varphi}{\partial x}\right) \psi_{+} \\
i\left(-2 \bar{t} a \frac{\partial}{\partial x}-\Delta\right) \psi_{+} & =\left(E+\bar{t} a \frac{\partial \varphi}{\partial x}\right) \psi_{-},
\end{aligned}
$$

which then allows one to straightforwardly generate a second-order differential equation for either $\psi$.

It is instructive to consider first the relatively simple case of a purely ionic ordered compound exhibiting no dimerization, so that $t=\partial t / \partial x=0$, while $\bar{t}=$ const. In this case, one obtains a Klein-Gordon equation for $\psi_{+}$:

$$
\left(-4 \bar{t}^{2} \frac{a^{2} \partial^{2}}{\partial x^{2}}+\varepsilon^{2}-2 \bar{t} a \frac{\partial \varepsilon}{\partial x}\right) \psi_{+}=E^{2} \psi_{+},
$$

and an analogous equation for $\psi_{-}$, not shown.

Generally, however, one obtains a more complicated equation, which can be interpreted as a Klein-Gordon equation with the energy reference point shifted off the classical vacuum state:

$$
\left(-4 \bar{t}^{2} \frac{a^{2} \partial^{2}}{\partial x^{2}}+\Delta^{2}-2 \bar{t} a \frac{\partial \Delta}{\partial x}\right) \psi_{+}=\left(E+\bar{t} a \frac{\partial \varphi}{\partial x}\right)^{2} \psi_{+}
$$

and an analogous equation for $\psi_{-}$, not given.

In going from Eq. (29) to Eq. (32), we omitted the following contribution to the l.h.s.:

$$
-2 a^{2} \frac{\partial \bar{t}^{2}}{\partial x} \frac{\partial \psi_{+}}{\partial x}+\frac{2 \bar{t} a\left(\bar{t} a+\Delta \psi_{+}\right)}{(E+\bar{t} a \partial \varphi / \partial x)^{2}} \frac{\partial}{\partial x}\left(\bar{t} \frac{\partial \varphi}{\partial x}\right) .
$$

The first term will contribute in the forth order in $k^{4}$ because the quantity $\left(\partial \vec{t}^{2} / \partial x\right)$ has a random sign. This circumstance will also automatically take care of the term itself being anti-hermitian. A contribution of order $k^{4}$ would exceed the accuracy of the long-wavelength approximation that led to Eq. (25) in the first place, hence we have omitted it. The second term can be analyzed analogously. For these same reasons, importantly, the quantity $\bar{t}^{2}$ in front of the second derivative in Eq. (32) should be regarded as a constant (though not necessarily equal to its average value!).

Apart from the "shift" term in the square brackets, Eq. (32) is well known,, 46.47 of course, as part of continuum descriptions of topological midgap states in conjugated polymers $\underline{4}$ and midgap states in amorphous chalcogenide alloys $\frac{48,49}{}$ In those descriptions, the electronegativity variation was considered as built-in and constant, as pertaining to a set of ions are rigidly assigned to the lattice. Here, we consider $\varepsilon$ as spatially varying to include the possibility of local polarization fluctuations. We observe that upon including this possibility, our "Klein-Gordon" equation is no longer an eigen-value problem. In fact, one anticipates that for sufficiently large values of fluctuations, the "electron" and "hole" (or "positron") solutions of Eq. (32) will mix implying an insulator-to-metal transition, owing to polarization fluctuations. This is consistent with the mechanism of the emergence of excitonic insulator ${ }^{50}$ states and is an encouraging sign indeed.

Equations analogous to Eqs. (32) and (31) can be written for the spatial coordinates $y$ and $z$ just as well. Added together, these equations yield an analogous equation to Eq. (10). Indeed, for small deviation of the energy from the (stabilized) bottom of the respective mobility band, call it $E_{c}$, Eq. (32) becomes linear in the energy $E$. Upon a change of variables $E \rightarrow E+E_{c}$, leaving in only the linear term in $E$, and noting that $E_{c} \sim \Delta$, we obtain that the magnitude of the fluctuation of the random potential goes with the quantity $\Delta / 2$ plus the (numerically comparable) contributions of the $\bar{t} a \partial \Delta / \partial x$ term in Eq. (32). These quantities are straightforwardly related to the local variation of the bond strength and electronegativity, c.f. Eqs. (24) and (27). Inasmuch as the distributed potential in Eqs. (32) and (31) change approximately linearly with the inter-atomic distances within the appropriate range, $V_{\text {rms }} \propto \delta r$, their fluctuations are approximately Gaussian, too. Thus we can carry over the predictions (12) and (13), due to Refs. 31 33, to the present context. Furthermore, the amplitude of those fluctuations should be proportional to that of a vibrating bond:

$$
V_{\mathrm{rmp}} \sim V \frac{\delta r}{a},
$$

where $\delta r$ is the typical vibrational amplitude:

$$
\frac{\delta r}{a} \leq\left.\frac{\delta r}{a}\right|_{T=T_{m}} \simeq 0.1
$$

The latter approximate equality expresses the Lindemann criterion of melting, by which the relative vibrational displacement is universally about one tenth near the melting temperature $T_{m} \stackrel{51,52}{\underline{5}}$ The energy parameter $V$ corresponds to the variation of the (local) band gap with bond deformation corresponding to long wavelength optical phonons, as just discussed:

$$
V \simeq \frac{\Delta}{\partial \ln r},
$$

where $r$ is the bond length, as in Eq. (16). We anticipate that $V$ is numerically close to several $\mathrm{eV}$. Indeed, according to Eq. (27) and the discussion following Eq. (16), fluctuations in the quantities $t_{1}$ and $t_{2}$ should contribute together a few $1 \mathrm{eV}$ for chalcogenides. We expect a comparable contribution from fluctuations in $\epsilon$. There will be some additional enhancement because of space dimensionality is three; Eq. (27) is for a 1D chain.

In view of the standard result ${ }^{53}$

$$
\delta r^{2}=\frac{\hbar}{2 m \omega} \operatorname{coth}\left(\frac{\hbar \omega}{2 k_{B} T}\right),
$$

relation (34) justifies the functional forms in Eqs. (2) and (4) used for the temperature dependences of the 
band gap and the width of the Urbach tail. We show the corresponding fits of the band gap for the substances shown in Fig. 1. alongside the experimental data. For the purpose of fitting, we have adopted the size of the rigid molecular unit - often called the "bead" - for the length L. By construction,,$\underline{54}$ motions on lengthscales greater than the bead size, the liquid behaves as that composed of weakly interacting particles, such as in Lennard-Jones systems. This is very much consistent with the definition of $L$ in Eq. (11). Ordinarily, the bead contains several atoms. We took the bead size for quartz from Ref ${ }^{54}$ : $L=3.6 \AA$; the bead essentially corresponds with the $\mathrm{SiO}_{4 / 2}$ unit. Likewise for the chalcogenides, we used the argument from Ref $\underline{49}$ that the bead corresponds to the $\mathrm{AsS}_{3 / 2}(L=3.9 \AA)$ and $\mathrm{AsSe}_{3 / 2}(L=4.1 \AA)$ pyramids. Explicit knowledge of the mass $m$ from Eq. (37) is not needed since the value of $\delta r / a$ is calibrated so that it achieves exactly $1 / 10$ at the melting temperature $T_{m}$. The latter values are listed in the caption of Table $\llbracket$.

The resulting values of the fitting parameters $V$ and $\omega$ from Eq. (37) are provided in Table \) they are very much in accord with expectation. The parameter $V$ for quartz is large than for the chalcogenides, which is consistent with its larger band gap.

The mapping between the present model and that from Refs. 3133 is far from exact. The auto-correlation of the noisy potential depend on the Cartesian distances between distinct locations. Yet for non-interacting chains, such correlations will be found only for pairs of points located on the same chain. Still, the chains must interact because of $s p$-mixing (or its analogs, if other types of orbitals are involved), various interactions among electrons and ions, and the for the simple reason that the lengths of distinct bonds emanating from an atom change all at the same time as the atom moves about. As a result, the random potential in the 3D version of Eqs. (32) and (31) will no longer be separable into functions of individual coordinates $x, y$, and $z$. Instead, the auto-correlation function for the fluctuations will be depend on the Cartesian distances between distinct locations, at least for not too long distances. In any event, we do not expect the predictions of the random-potential model to depend much on the precise spatial shape of the potential well of size $L$ so long as the well itself has sufficiently steep walls, see discussions in Refs. 32 and 33 .

We observe that at this level of approximation, the fluctuations of the random potential are coupled to lattice motions that modify the differentials in the transfer integral and electronegativity largely within the unit cell, see Eqs. (18) and (19), not between distinct cells. These are motions that correspond to optical phonons. More formally, we note that the potential energy terms in Eqs. (32) and (31) are largest in the center of the Brillouin zone but become completely suppressed at the edge of the zone. (We remind the reader that Eqs. (32) and (31) were obtained by expanding around that edge.) Likewise we note the interaction with acoustic phonons near the edge of the Brillouin zone, which is represented by the first term in Eq. (33), does not contribute to the random potential at this level of approximation. Such contributions are however expected closer to center of the Brillouin zone. For instance, one expects that thermal expansion accounts in part for the narrowing of the band gap $\stackrel{10}{\underline{10}}$

We next comment on the requirement of the model from Refs. 3133 that noise-autocorrelation be very strong within a certain distance, $L$, but decay very rapidly beyond that, as in Eq. (11). That the decay should be so rapid is somewhat puzzling since we expect spatial correlation in solid to decay no faster than the screened Coulomb interaction, if that fast. At the same time, the parameter-free calculation of Grein and John, Ref. 14 and references therein, reproduces the gross features of the Urbach tail quantitatively at sufficiently high temperatures, while indicating that non-adiabatic effects are important, at least for crystalline materials. One may elaborate on these notions in the present context by first reiterating that the random-potential model treats the nuclear motions entirely classically. On the other hand, it is not guaranteed that the local vibrational configurations corresponding to specific local fluctuations of the random potential would be in quantum-mechanical resonance. Accounting for the presence/lack of such resonances is routinely done in theories of non-adiabatic charge transfer $\stackrel{55}{\underline{5}}$ Hereby, one finds the rate of charge transfer or optical absorption by directly summing over the spectra of the two sets of lattice-vibrational modes corresponding, respectively, to the initial and final states of the transferred electron. As a result, the charge transfer rate is modulated by wave-function overlaps between these two sets, i.e., the Frank-Condon factors.

We next outline how to estimate these Franck-Condon factors using the present picture. First we note that the spring constant $\kappa$ of a bond is correlated with the bond strength,,$\frac{56}{5}$ hence one can write qualitatively:

$$
\kappa \propto t_{\mathrm{e}}^{\alpha},
$$

where the transfer matrix $t_{e}$ is from Eq. (16) and $\alpha$ is some positive power, see also Section $\nabla \nabla$ Because of the close association between the bond strength and its stiffness, we expect that to a localized electron, there will correspond a set of localized vibrations as well. This can be seen relatively directly as follows.

The vibrational spectrum of a chain corresponding to the electronic spectrum from Eqs. (17)-(19) is similar to the electronic spectrum, with the difference that its ground state is at zero frequency, of course, while the gap between the acoustic and optical branch spans the vibrational frequency of the weak and strong bond, respectively, if the masses of the ions are the same $\frac{57}{5}$ When the ions masses are different, the situation is a bit more complicated. Consider the following, 1D vibrational Hamiltonian:

$$
\mathcal{H}_{\mathrm{vibr}}=\sum_{n} \frac{m_{n}}{2} x_{n}^{2}+\frac{\kappa_{n, n+1}}{2}\left(x_{n}-x_{n+1}\right)^{2},
$$


where both the ion mass and the spring constant strictly alternate in magnitude from site to site:

$$
\begin{gathered}
m_{n}=\left\{\begin{array}{l}
m_{1}, n \text { odd } \\
m_{2}, n \text { even }
\end{array}\right. \\
\kappa_{n, n+1} \equiv \bar{\kappa}+(-1)^{n} \kappa, \quad|\kappa| \leq \bar{\kappa} .
\end{gathered}
$$

In this case, the vibrational eigenfrequency at wavevector $k$ is determined by solving the characteristic equation for the following matrix:

$$
\left(\begin{array}{c|c}
-m_{1} \omega^{2}+2 \bar{\kappa} & -2 \bar{\kappa} \cos k a+2 i \kappa \sin k a \\
\hline-2 \bar{\kappa} \cos k a-2 i \kappa \sin k a & -m_{2} \omega^{2}+2 \bar{\kappa}
\end{array}\right),
$$

c.f. Eq. (22.35) of Ashcroft and Mermin 57 and Eq. (22). Upon introducing the harmonic average of the ion mass:

$$
m=\sqrt{m_{1} m_{2}},
$$

one can re-trace the derivation of Eq. (25) to obtain the vibrational analog of the continuum Hamiltonian which determines the eigenvalues for the quantity $m \omega^{2}$ :

$$
\mathcal{H}=-i 2 \bar{\kappa} a \hat{\sigma}_{3} \frac{\partial}{\partial x}-2 \kappa(x) \hat{\sigma}_{1}-\frac{\Delta m}{m} \bar{\kappa}(x) \hat{\sigma}_{2}+\frac{M}{m} \bar{\kappa},
$$

where

$$
\begin{aligned}
\Delta m & \equiv m_{1}-m_{2} \\
M & \equiv m_{1}+m_{2}
\end{aligned}
$$

and we allow for slow variation in the bond strength. One can make the Hamiltonian (44) of dimensions energy by multiplying it by $a^{2}$. Analogously to Eq. (25), Hamiltonian (44) gives the energy per atomic site.

Although the correspondence between the electronic and phonons Hamiltonians is not exact - far from it - the similarity is hard to miss. Note that the mass differential between the two distinct ions plays a formally similar role for the vibrational spectrum to the role the electronegativity variation plays in the electronic spectrum. In contrast with the electronic spectrum, the vibrational spectrum is coupled not only to long wave-length optical distortions, as embodied in the $2 \kappa(x) \hat{\sigma}_{1}$ term in Eq. (44), but also to the acoustic modes, via the term $(\Delta m / m) \bar{\kappa}(x)$.

Localized vibrations are modes whose frequencies fall within the forbidden gap between frequencies $\omega_{v}$ and $\omega_{c}$ in Fig. 3. The behavior of vibrationally localized solutions can be understood already from the simplest version of Hamiltonian (44), in which the average force constant and its alternation pattern is spatially homogeneous. The resulting Klein-Gordon equation for the vibrational eigen-modes is

$$
\left(-4 \bar{\kappa}^{2} \frac{a^{2} \partial^{2}}{\partial x^{2}}+\Delta_{v}^{2}\right) \psi=\left(m \omega^{2}-M \bar{\kappa} / m\right)^{2} \psi
$$

where $\Delta_{v}$ is the vibrational gap:

$$
\Delta_{v} \equiv\left[(2 \kappa)^{2}+(\bar{\kappa} \Delta m / m)^{2}\right]^{1 / 2} .
$$

The edges of the vibrational gap from Fig. 3 are given by the expressions $\omega_{c, v}^{2}=M \bar{\kappa} / m^{2} \pm \Delta_{v} / m$. The maximum achievable phonon frequency is

$$
\omega_{D}^{2}=2 M \bar{\kappa} / m^{2}=2 \bar{\kappa} / \mu,
$$

where $\mu \equiv m_{1} m_{2} / M$ is the reduced mass of the bond.

According to Eq. (47), a localized vibrational mode centered at $x_{0}$, would exponentially decay away from $x_{0}$ :

$$
\psi(x) \propto e^{-\left|x-x_{0}\right| / \xi_{v}},
$$

where

$$
\xi_{v} \equiv a \frac{2 \bar{\kappa}}{\Delta_{v}}=a\left(\frac{\kappa}{\bar{\kappa}}+\frac{\Delta m}{m}\right)^{-1} .
$$

This is entirely analogous to how an edge electronic state or midgap electronic state, in the context of Eqs. (25) and (32), would decay exponentially rapidly into the bulk $, 44,47,48$ See also a pedagogical discussion in Ref. 42 . Likewise, one can think of the vibrational localization length $\xi_{v}$ as the penetration length of an instanton.

To assess the aforementioned Franck-Condon factor, we make the following mental construct: Imagine an instantaneous aperiodic configuration of the ions that lead to both a localized electronic solution and, by virtue of Eq. (38), to a localized vibrational mode. The two localized modes will be centered on the same site and decay exponentially into the bulk, as just mentioned. The Franck-Condon factor corresponding to the electron shifted from location $x_{1}$ to $x_{2}$ is proportional to

$$
e^{-\left|x_{1}-x_{2}\right| / \xi_{v}} \prod_{n} e^{-\left(x_{n}^{(1)}-x_{n}^{(2)}\right)^{2} / 2 \delta x_{v}^{2}},
$$

where $x_{n}^{(1)}$ and $x_{n}^{(2)}$ denote the position of atom $n$ corresponding to the electronic configurations 1 and 2, respectively. The quantity $\delta x_{v}$ is the amplitude of the localized vibrational mode. The vibrational frequencies in question are within the gap and thus intermediate in value between the acoustic and optical branch, i.e., roughly half the Debye frequency. Thus $\delta x_{v}$ exceeds its zero-point value but not by much. Hence one may write:

$$
\delta x_{v} \sim\left(2 \hbar a / \xi_{v} M \omega_{D}\right)^{1 / 2},
$$

Here we estimated the mass of the mode by counting the ions within the correlation length $\xi_{v}$. In view of Eq. (38), $\xi_{v}$ will be comparable to the electronic instanton length, which is ordinarily of the order 10 lattice spacings in amorphous semiconductors $\stackrel{48,49}{,}$ but could become quite large when the forbidden gap becomes small, see Eq. (51).

Because of this large mass, the vibrational amplitude $\xi_{v}$ will be very small, significantly smaller than the vibrational amplitude of an individual atom. This implies that 
the product of individual Franck-Condon (FC) factors in Eq. (52) will be very very small except, perhaps, in the case of strict periodicity of the lattice. (Note the FC factors for atoms far away from the localized mode are numerically close to one; the said smallness is largely due to the atoms contributing to the localized mode.) The small magnitude of the FC factor can thought of as the motion of the electron being virtually recoil-less when it is subjected to a fluctuating potential. (This lack of recoil on the part of the lattice is reminiscent of the Mössbauer effect.) This, then, suggests that in an internally consistent treatment, auto-correlation of the random potential should decay exceptionally fast, by construction. This is consistent with the postulated rapid decay from Eq. (11). Once the correlation does begin to decay, it should do so like the Franck-Condon factor in Eq. (52), i.e., in a Gaussian fashion. Once again, the large mass of the localized vibrational mode is what renders the classical treatment of the potential fluctuation from Refs $\frac{31-33}{-}$ while assuming short-range correlations - internally consistent.

Finally note that the possibility of a somewhat slower decay in the case of strict periodicity seem to be consistent with the Urbach tail in crystalline Si exhibiting some structure $\underline{14}$ No such structure is expected in amorphous materials, as already mentioned.

\section{THE INTRINSIC RELATIONSHIP BETWEEN ELECTRONIC EXCITATIONS ACROSS THE BAND GAP, OPTICAL PHONONS, AND METALLICITY}

Thus we have argued that in compounds of Type 1 and 2, long wavelength optical phonons are intrinsically related to and can quantitatively account for the stabilization of the band gap and, at the same time, the appearance of the Urbach tail. The two types of compounds can be understood as supporting a standing charge density wave whose crests are centered, respectively, on bonds and ions, respectively. Significantly, the CDW must have the exact same symmetry with respect to translations as the lattice itself. The two types of CDW are mathematically equivalent, see Eq. (25), but generally result from very different physical processes. To give rise to an off-center, bond-order CDW, the atoms would have to move distances intermediate between the atomic vibrational amplitude, $\sim a / 10$ and atom spacing, $a$. An example of such a process is the simple-cubic to rhombohedral arsenic transition ${ }^{58,59}$ For an on-site CDW, on the other hand, two following possibilities come to mind: On the one hand, such a CDW can be created by placing two atoms with differing electronegativies in the alternating positions on a bi-partite lattice such as in the $\mathrm{NaCl}$, $\mathrm{CsCl}$, wurzite, or zincblende lattice. In addition, a spontaneous polarization can be envisioned, such as during charge disproportionation, $\underline{\underline{60}}$ which may or may not be accompanied by a displacive transition.

The apparent connection between the presence of the

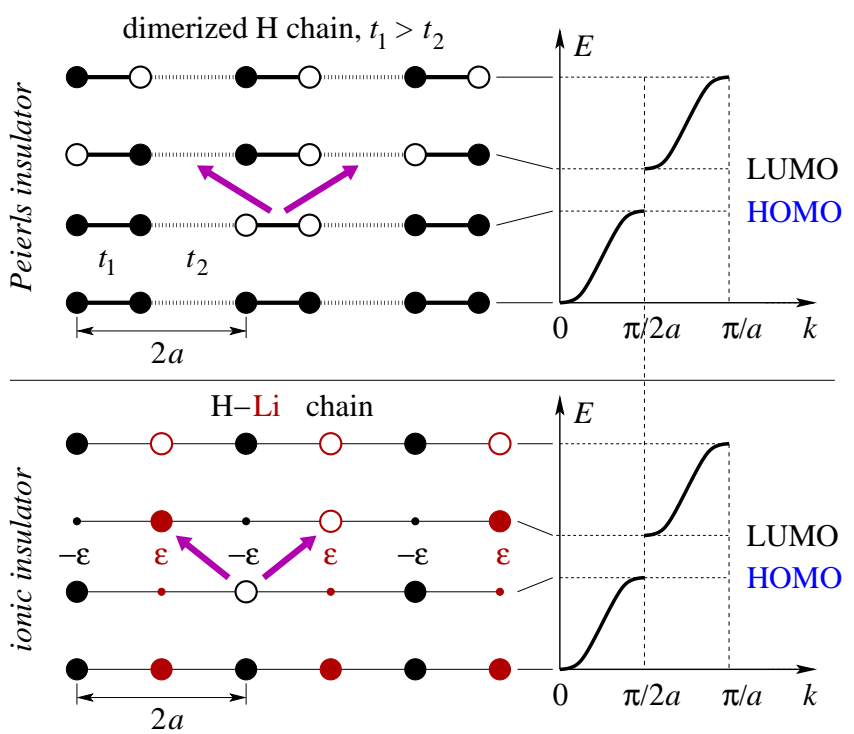

FIG. 4. A molecular orbital (MO) representation of the wavefunctions corresponding to the band edges in a 1D insulating chain. The top and bottom panel correspond with, respectively, Type 1 and 2 insulators. (In the Type 1 case, we adopted the extreme case $\varepsilon=0$.) Filled and empty circles correspond with opposite sign of the wavefunction, on the respective site. The size of the circle reflects the local magnitude of the wavefunction. The arrows show how the charge is transferred during a HOMO $\rightarrow$ LUMO excitation.

optical gap, on the one hand, and optical phonons, on the other hand, can be discussed using already simple notions of the molecular orbital (MO) theory. The band edge MOs for compounds of type 1 and 2 are sketched in Fig. 4. top and bottom panels, respectively. There, one can trace local polarization - spontaneous or that arising upon optical excitation - to movements of either the lobes or nodes in the corresponding electronic wavefunction. Clearly, such local polarization patterns correspond to an optical phonon.

Compounds of Type 3 may seem to not fit in the present formalism at a first glance. Consider, however, crystalline silicon or germanium, which adopt the diamond lattice at normal conditions. This lattice has two atoms in its primitive cell. The respective sets of these two equivalent atoms can be thought of as each forming equivalent FCC sub-lattices. (The two FCC sub-lattices are shifted relative to each other along the main diagonal of the cubic unit cell, by a quarter of the length of the diagonal.) The primitive cell of the diamond lattice is the same as that of either of the FCC sub-lattices. The overall electronic structure can be thought of, even if somewhat unconventionally, as a resonance between two sets of atomic orbitals centered, respectively, on the two FCC sub-lattices. Yet each of these sets corresponds to a charge density wave that has the same primitive cell as the lattice itself. Thus the valence and conduction band 
of Si can be thought of, respectively, as the bonding and anti-bonding combination of two CDW states. Similarly to the simple picture in Fig. 4. excitations across the gap or spontaneous polarization will involve charge redistribution within the primitive cell of the lattice - and hence coupling to optical phonons-because an anti-bonding orbital has an extra node compared with the corresponding bonding one. The so locally-polarized state would have the same symmetry with respect to translations as the lattice itself, just as it was for materials of Type 1 and 2. (We anticipate the detailed pattern of local polarization could be a quite more complicated than that sketched in Fig. 4 because the constituent CDW states are themselves complicated. Consistent with this notion, silicon and electronically similar materials have an indirect gap.)

Conversely, suppose there exists an insulator that has just one atom per primitive cell. The preceding discussion suggests that excitations across the gap could not create a local-polarization pattern that has the same translational symmetry as the lattice. This would be fine for a solid made of closed shell atoms: For instance, optical transitions can be simply thought of as optical excitations of weakly-interacting individual atoms. Consistent with this, elemental helium metalizes only at high pressures. 61 However in the presence of covalent bondingi.e. bonding between partially filled atoms shells - this picture seems potentially problematic because every valence atomic orbital is strongly hybridized with the orbitals centered on neighboring atoms, by the very definition of covalent bonding. It stands to reason that optical excitations would create a polarization pattern in a covalently bonded solid. Yet the pattern would have to have a lower translational symmetry than the lattice itself. Thus polarization-induced stabilization and broadening of the band edge, if any, would be much weaker - despite the molecular orbitals being delocalized!- suggesting the supposition that our insulator has just one atom per primitive cell was internally-inconsistent.

To make the above notion more systematic, we first recall a formal, yet fundamental aspect in which insulators are different from metals: First imagine a set of molecular orbitals (MOs) that diagonalize an effective one-electron Hamiltonian. The totality of the MOs form a complete basis set and so the atomic orbitals can be recovered from the MOs by a suitable unitary transformation. Now suppose the MOs are not all filled, as would be the case if the participating atoms are not all closed-shell. The filled MOs alone no longer comprise a complete set. Thus, the Mullican-Hund (MO) and Heitler-London (localized) descriptions are not equivalent unless all MOs are filled, as emphasized by Burdett. 62,63 This elementary notion naturally leads to a more general observation that the band description and the description in terms of localized Wannier orbitals are equivalent only in insulators. In fact, it was shown relatively recently that there do exist exponentially localized Wannier orbitals for 2D and 3D insulators ${ }^{64}$ On an historical note, localized-MO formalisms have attracted attention of physicists and chemists alike over the years, $, 39,65-69$ despite their non-uniqueness. Perhaps the most immediate use of localized Wannier-like orbitals is to represent complicated results of quantumchemical calculations so as to appeal to the concepts of the canonical two-center bond, closed shell interactions, and lone pairs. Conversely, this formalism helps highlight situations where bonding becomes truly multi-center $\underline{39}$

Now imagine a covalently bonded insulating solid made of partially filled atomic orbitals. For the sake of completeness, assume there is one filled and one empty band. The orbitals comprising the filled band can be represented in terms of mutually-orthogonal Wannier orbitals, call them the "electron Wannier orbitals." Likewise, the orbitals comprising the empty band can be represented in terms of mutually-orthogonal "hole Wannier orbitals." The charge distribution pertaining to the electron Wannier orbitals(but not the individual orbitals!) must have the symmetry of the lattice with respect to translations, and likewise for the hole Wannier orbitals. At the same time, the electron and hole Wannier orbitals are not identical; instead, they are mutually complimentary. The former set is made of the bonding orbitals, and the latter set of the anti-bonding orbitals. Thus we conclude that in a covalently bonded insulator, excitation across the forbidden gap must result in a polarization pattern that has the same symmetry with respect to translations as the lattice itself. At the same time, the charge redistribution that leads to this polarization pattern must involve charge transfer between atomic sites and/or interatomic spaces, analogously to the specific examples considered in the beginning of this Section. Indeed, the Wannier orbitals are not the same as the atomic orbitals. Already the number of the former is less than that of the latter. Thus a Wannier orbital covers more than one atomic orbital. Furthermore, at least a subset of the Wannier orbitals in a covalently bonded system must include combinations of orbitals centered on distinct atoms, as opposed to orbitals centered on the same atom. To drive this notion home we recall that the Wannier orbitals are linear combinations of the occupied (vacant) MOs, not the atomic orbitals themselves. The latter, informally speaking, are not even accessible by themselves but only in bonding (anti-bonding) combinations of atomic orbitals centered on distinct atoms. Many specific examples of such localized orbitals can be found in the literature $39,65,66,69-71$

Thus we conclude that in a covalently bonded crystalline insulator, the polarization pattern resulting from an excitation across the band gap can have the symmetry of the lattice itself and, at the same time, involves charge transfer between distinct atoms and/or interatomic spaces. Thus the primitive cell of a covalently bonded insulator must contain at least two distinct atoms and thus should exhibit optical phonons. In the case of ionic or aperiodic solids, this is automatically true, of course. (We note that liquid insulators would be classified as amorphous solids because electrons move much faster than nuclei.) A corollary of this observation is that 
if a solid lacks optical phonons, it must be a metal. Ordinarily, a solid lacking optical phonons has no more than one atom per primitive cell (but see below for additional discussion). These notions are consistent with experience: For instance, browsing through the Periodic Table reveals that every elemental solid that is not metallic has at least two atoms per primitive unit. In some cases, we have covalently bonded solids such as silicon or germanium; the diamond lattice has two atoms per primitive cell. In the case of halogens or nitrogen, for instance, the solids can be thought of as molecular solids made of closed-shell dimers. Either way, that these molecular solids are insulating is consistent with the present discussion. To avoid confusion we note that the reverse of this conclusion is generally not true: The presence of optical phonons does not preclude the solid from being metallic.

Although the above argument was stated using atomic orbitals as a local basis set, it can be reformulated without explicit reference to atomic orbitals but, instead, only using distinct sites in space. This would be useful to systems such as Wigner solids, in which the counter-charge is distributed uniformly in space by construction and so there is no obvious set of atomic-like basis set functions, see below. Stated more generally, the preceding conclusion can be stated as follows: The primitive cell of an insulating solid should contain at least two spatially inequivalent localized basis orbitals. This could be realized in a variety of ways. When atoms are indeed present, one can imagine a solid with just one atom per primitive cell but a CDW that has a lower symmetry. Imagine for instance a bcc lattice made of identical ion cores that hosts an on-site CDW whose symmetry is lower, namely, that of the $\mathrm{CsCl}$ lattice. The latter lattice is bi-partite, of course, consisting of two equivalent simple cubic lattices. Likewise, an elemental solid exhibiting the simple cubic structure would have to be metallic but could become insulating upon emergence of a CDW whose crests and troughs each form the rock salt structure; the latter represent a bi-partite lattice made of two fcc sub-lattices. One can also imagine various distortions of the above lattices, such as tetragonal or monoclinic, that do not lead to an increase in the number of atoms per primitive unit.

Such symmetry-lowered bi-partite solids would host at least three optical phonon branches even though the original ions comprise a higher symmetry lattice. X-ray or electron scattering would pick up this lowering of the symmetry (unless the CDW is dynamic on the time scale of the experiment). In this sense, these solids should not be regarded as containing only one atom per primitive cell despite the ionic cores comprising the sub-lattices of lower symmetry being identical. Instead, one has a symmetry breaking in the way of charge disproportionation that effectively leads to the appearance of two chemically distinct species. We are not aware of charge disproportionation in the form of simple bi-partite lattices in elemental compounds, however more complicated charge disproportionation patterns have indeed been observed in the form of host-guest structures $\underline{\underline{59}}$ The emergence of such patterns does not seem to affect the fact of metallicity of the material. Perhaps bi-partite like charge disproportionation patterns could be observed at sufficiently high pressures. In this regard, studies on the high pressure behavior of hydrogen come to mind ${ }^{72}$ These studies suggest that solid hydrogen would become metallic before its structure would be so symmetric as to contain only one atom per primitive cell, see also Ref. 73. No charge dispropotionation seems to occur.

We have mentioned the zincblende and wurzite lattices as examples of standing charge density waves. Here we note that their "parent lattices," i.e. the the diamond and lonsdaleite lattices, respectively, for instance, already have two atoms per primitive cell and do not have to be metallic.

Even in the absence of charge disproportionation, the symmetry can still be lowered by the emergence of spindensity wave (SDW). This symmetry lowering would not be picked up by X-ray diffractometry, but could be detected by neutron scattering (unless the SDW is dynamic on the time scale of the experiment.) We do not expect that a spin-density wave by itself would cause the material to exhibit optical phonons let alone become insulating. For instance, chromium is known to spontaneously develop an SDW at low temperatures - both commensurate and incommensurate $\stackrel{74}{ }$ As a secondary effect stemming from electron-phonon interaction, a strain wave $\frac{75}{}$ and a CDW 76 emerge alongside as well. Neither of these lead to the disappearance of the metallicity. In discussing purely electronic mechanisms to lower the symmetry of the lattice, we should mentioned that, in the first place, the emergence of charge/spin density wave or any kind of charge disproportionation could lead a more basic kind of symmetry breaking, namely, in a way of static lattice distortions 60 Perhaps the simplest example of such a symmetry broken solid is the rhombohedral arsenic,, 58 which can be thought of as 3D analog of a Peierls insulator ${ }^{77}$ In the case of such displacive transitions, optical phonons will emerge automatically. An insightful way to connect various lattice distortions with the underlying electronic phenomena is through notions of reduction of dimensionality, $\stackrel{78}{ }$ again consistent with the notion of the metal-insulator transition as a symmetry lowering.

Yet in some cases a spin-density wave could be in fact indicative of substantial - even complete - electronic localization. According to existing studies, a periodic Wigner solid in 3D should be bcc $\stackrel{79,80}{ }$ The Wigner bcc solid has just one site per primitive unit implying it must be metallic even at absolute zero. This would seem to contradict the premise of the electron assembly being a solid, in which the electrons are strictly localized. The latter point deserves some elaboration. First we note that the reported phase diagrams are most likely oversimplified in that they show a sharp transition line between the liquid and solid phase in the temperature-density plane. Yet the transition must be discontinuous except, perhaps in one isolated point $\stackrel{81-84}{-84}$ Thus, properly, those phase diagrams should show a substantial coexistence region, 
which physically corresponds to macroscopic parts of the system being occupied by the solid and liquid phase, respectively. This means that the reported transition density is likely higher than the density of crystal and lower than the density of the liquid that would actually spatially coexist. (The reported transition itself probably corresponds with mechanical, not thermodynamic melting $\underline{51}$ ) Now, imagine approaching the solid-to-liquid transition from the crystal side, all at $T=0(!)$, and suppose for a moment that instead of spatial coexistence, the appearing liquid is uniformly distributed over the sample. (This would imply the solid is metallic.) Clearly such uniform liquid would be of much lower density than required for its stability. Hence, the crystal and the liquid cannot coexist in the same region but only distinct regions in space. This notion adds to the observation due to Golden et al $\stackrel{39}{\underline{3}}$ who have suggested electronic fluids with differing degrees of localization would not mix at sufficiently high density. To avoid confusion we note that at finite temperatures, some of the electrons are allowed to be promoted to the conduction band subject to activation across the forbidden gap. The density of this (thermally activated) electronic fluid could be made arbitrarily small since it is not in bulk equilibrium with the solid.

At the same time, the possibility of the Wigner crystal hosting a CDW with the translational symmetry of the $\mathrm{CsCl}$ lattice can be excluded because of quantum fluctuations: The system would readily minimize its kinetic energy by assuming a superposition of chargedisproportionated configurations.

The only remaining way to lower symmetry so as prevent the primitive unit to have more than one inequivalent site is to have an antiferromagnetic (AF) ordering. This is consistent with the results of quantum Monte Carlo simulations $\frac{16}{\underline{16}}$ In $3 \mathrm{D}$, the simplest kind of $\mathrm{AF}$ ordering could be realized by having the Cs sites in the $\mathrm{CsCl}$ lattice to be polarized up and $\mathrm{Cl}$ sites polarized down, for instance. In 2D, such anti-ferromagnetic ordering could be realized by an alternating sequence of chains polarized up and down. $\underline{17}$

In a distinction from insulating solids made of atoms, the Wigner solid exhibits only three phonons branches, two of which are gapless and correspond with the transverse acoustic phonon branches of a regular solid; neither incur local density changes. On the other hand the longitudinal mode, which represents the plasma oscillations, is gapped. The gap comes about because longitudinal sound waves do incur density changes while the Coulomb forces are long range thus imposing a finite energy cost even on the longest wavelength vibrations $\underline{52}$ The plasmon can be thought of as a Higgs boson $\underline{85,86}$

\section{METAL-INSULATOR TRANSITION AS A SYMMETRY BREAKING}

We have discussed manifestations of an intrinsic connection between the presence of a band gap and optical phonons. Here we wish to take a more general perspective on this connection. The dimerized chain, such as that depicted in Fig. 4, is clearly of lower symmetry, with respect to translation, than the original nondimerized chain. But at the electronic level, the symmetry breaking is actually a bit more interesting. Indeed, consider the $k=\pi / 2 a$ orbitals centered on the odd- and even-numbered sites from Eq. (20), call them $\psi_{1}$ and $\psi_{2}$. These have the same energy if $t=\varepsilon=0$. Any pair $\psi_{1} \cos (\phi / 2)+i \psi_{2} \sin (\phi / 2)$ and $i \psi_{1} \sin (\phi / 2)+\psi_{2} \cos (\phi / 2)$ of these two orbitals would form an equivalent set of solutions as well, for any value of $\phi$. Yet this (continuous) symmetry with respect to $\phi$ is broken following the dimerization. For instance, for the "Peierls insulator" in Fig. 4, $\varepsilon=0$ the value $\phi=\pi / 2$ is selected, as is easy to see from Eq. (22). The ionic case, on the other hand, selects out $\phi=0$.

There are other ways to look at this symmetry breaking: The optical phonons can be thought of resulting from chemical ordering, in the form of a specific arrangements of ions or bond strength. To restore the symmetry in the latter case, the ions or bonds of differing strength would have to be able to swap places, which would require some nuclear motions, too. The strength of the CDW appears to be an appropriate order parameter to describe the symmetry breaking. Viewed this way, the optical phonons could be thought of as fluctuations of the order parameter of the metal-insulator transition. These notions prompt us to take a field-theoretic perspective on the discussion from the preceding Sections. As an additional benefit, a symmetry based discussion is expected to be relatively robust with regard to many-body effects.

We will build a density functional description in the spirit of Landau's analysis of the liquid-to-solid transition, 82 where the order parameter reflects the magnitude of a density wave near the wavelength corresponding to the particle spacing in the putative solid, see also Ref. 81 . It is most convenient, for the present purposes, to use the parameters $\varepsilon$ and $t$ from Eq. (25) as the order parameters. These can be regarded as the components of the molecular field ${ }^{81}$ that control entries of the electronic density matrix

$$
P_{\alpha \beta}=\sum_{s= \pm 1 / 2}\left\langle c_{\alpha, s}^{\dagger} c_{\beta, s}\right\rangle=2 \sum_{k, \text { occ }} \psi_{k \alpha} \psi_{k \beta}^{*},
$$

Specifically, the spatial variation of the on-site electronic density:

$$
\rho \equiv \frac{1}{2}\left(P_{2 n+1,2 n+1}-P_{2 n, 2 n}\right) .
$$

can be computed as the following derivative:

$$
\rho=-\frac{\partial E}{\partial \varepsilon} .
$$


Likewise, the variation in the bond strength

$$
\eta \equiv \frac{1}{2}\left(P_{2 n, 2 n+1}-P_{2 n, 2 n-1}\right)+\text { C.C. }
$$

is the conjugate variable to the transfer integral:

$$
\eta=-\frac{\partial E}{\partial t}
$$

By adopting the restrictions from Eqs. (18) and (19) we limit ourselves, by construction, to wavelengths near the period of the dimerized chain. To obtain a density functional theory in the sense of Kohn and Sham,,$\frac{87}{4}$, which would operate on the variables $\rho$ and $\eta$, one can perform a Legendre transform, $\frac{39}{3}$ something we will not do here.

Already a quick look at the spectrum in Eq. (23) shows that the energy $E$ of the non-interacting model, at halffilling, is maximized at $\varepsilon=t=0$ and monotonically decreases away from the maximum. Near $\Delta=0$, is expected to behave smoothly in spatial dimensions higher than one:

$$
E=\frac{a_{2}}{2} \Delta^{2}+\text { higher order terms, }
$$

where the coefficient at the quadratic term

$$
a_{2} \sim \frac{1}{W}
$$

is largely determined by the width $W$ of the valence zone. In $1 \mathrm{D}$, the coefficient $a_{2}$ is actually negative and singular $a_{2} \propto \ln \Delta^{2}, \underline{88}$ implying that irrespective of interactions, the chain will dimerize. Indeed, fixing $\varepsilon$ and adding a penalty for distorting the bonds, $\propto\left(+t^{2}\right)$ will produce a bistable potential energy surface, if $\varepsilon$ is not too large. The two minima correspond to the alternative ways to dimerize. In dimensions higher than one, the non-interacting part is no longer singular; the symmetry breaking may or may not take place depending on the geometry and interactions. For instance, electron-electron repulsion will generally suppress dimerization.

Above said, let us assume until further notice that the system does indeed exhibit a continuous symmetry breaking:

$$
a_{2}<0
$$

just like the non-interacting 1D models from the preceding Sections. Because the order parameter is vectorial, the broken symmetry is continuous, consistent with the elementary discussion in the beginning of this Section. Cases of broken continuous symmetry are interesting because they sometimes result in massless, Goldstone modes. Let us see that no such modes arise in the present case however.

First suppose the opposite, i.e., that the broken symmetry functional has the "mexican hat shape" sketched in Fig. 5] The Goldstone mode, if any, would correspond with fluctuations of the vectorial order parameter such

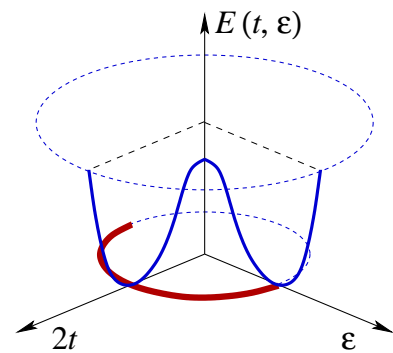

FIG. 5. A hypothetical, rotationally-symmetric free energy profile as a function of the strength of the charge-density wave, $\rho$ and $\eta$ standing for the on-site and off-site components, respectively. The semi-circle at the bottom of the potential exemplifies a hypothetical trajectory in the $(\rho, \eta)$ space for a transition between two distinct CDW states.

that its end moves along the local direction of the circular groove. In contrast with such low magnitude motions, one can also envision motions where the end of the vector moves a substantial amount. Such motions would correspond to a crossing of a domain wall separating equivalent symmetry-broken states, such as states with distinct polarizations of the Heisenberg magnet. In the present context, these distinct states would correspond to equivalent CDW states. Yet in the absence of magnetic field, self-generated or externally imposed, such a trajectory would be forbidden. Indeed, consider the Hamiltonian (25) where the $x$-axis is perpendicular to the domain wall and the vector $[2 t(x), \varepsilon(x)]$ performs a circular motion at a steady rate alongside: $\Delta(x)=\Delta_{0}, \partial \varphi / \partial x=k_{0}$. The sign of the constant $\varphi_{0}^{\prime}$ depends on the direction of rotation. This yields the following spectrum:

$$
E_{k}=-\bar{t} a k_{0} \pm \sqrt{\Delta_{0}^{2}+(2 \bar{t} a k)^{2}} .
$$

That is, the Fermi level shifts up or down depending on the sign of $k_{0}$. This shift contradicts the notion of the two directions of $\boldsymbol{k}_{0}$ being physically equivalent. Less obvious from the continuum solution is that a non-vanishing $k_{0}$ makes the system metallic, thus contradicting the original premise of the CDW being a gapped insulator. Indeed, for each additional "revolution" of the $(\varepsilon, 2 t)$ vector, a state is transferred between the valence and conduction band, the direction of transfer depending on the sign of $k_{0}$, see the solution of a discrete system in Fig. 6. There we also observe that additional, relatively narrow forbidden gaps may appear in the spectrum.

We note it is in principle possible to have a sense of direction for an interface in the CDW phase, if the time-inversion symmetry is broken. The latter would be the case, for instance, when (local) magnetic field is present. Such local magnetic fields could arise, for instance, in a suitably modified Hubbard model from vortex-like structures made of spins; the structures extend in a $2 \mathrm{D}$ plane $\underline{\underline{89}}$ When standalone, these so called meron vertices are infinitely-costly and thus end up forming strongly bound meron-antimeron pairs, similarly to extended dislocations. 


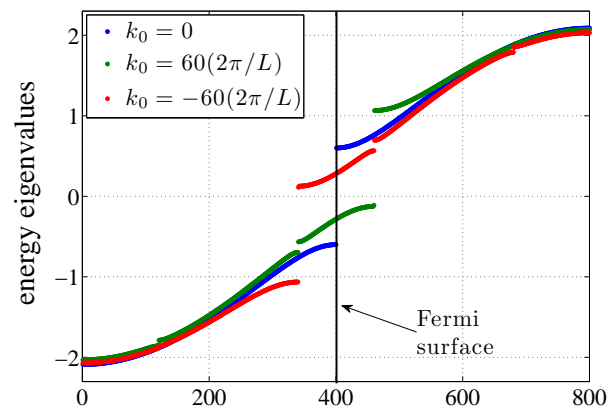

FIG. 6. The spectrum of the model (17) with a periodically varying $\varepsilon=\Delta_{0} \cos \left(k_{0} x\right)$ and $t=\left(\Delta_{0} / 2\right) \sin \left(k_{0} x\right) . \quad \bar{t}=1$, $\Delta=0.6, \mathrm{~N}=800$.

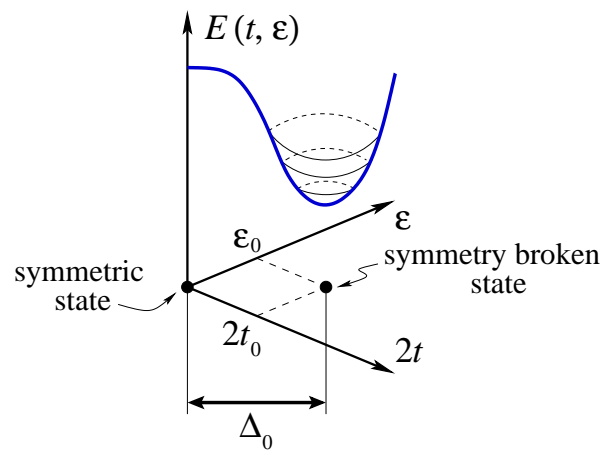

FIG. 7. Typical behavior of the optical absorption spectrum as a function of temperature. The width of the exponential tail of localized states is seen to change synchronously with the mobility edge.

We thus conclude that the free density functional can not have minima with vanishing curvature but, instead, should exhibit isolated minima separated by barriers. Such a minimum can be described, near its bottom, by a positively-defined quadratic form:

$$
E=\frac{\alpha_{1}}{2}\left(t-t_{0}\right)^{2}+\frac{\alpha_{2}}{2}\left(\varepsilon-\varepsilon_{0}\right)^{2},
$$

where for simplicity we assumed that the cross-term vanishes. (This can be always achieved by an appropriate rotation.) The (bulk) energy function is sketched in Fig. 7. Such a stable minimum can be obtained by using a higher order term with a positive coefficient in Eq. (59); a cubic or quartic term will ordinarily suffice. Simple algebra shows that the coefficients $\alpha$ are equal to $\left|a_{2}\right|$ times a numerical factor of order one:

$$
\alpha_{i} \sim 1 / W .
$$

A proper field theory is obtained by adding to Eq. 63. a penalty for spatial variation of the parameters $t$ and $\varepsilon$, for instance in the form of square gradient terms $K_{t}(\nabla t)^{2} / 2$ etc. The "spring constant" $K_{t}$ and its likes would be determined by the detailed interactions and geometry; they are complicated objects that that we have not discussed in this article. While the value of $t$ can be thought of as controlled by the propensity to displacive transitions, penalty for its spatial variation seems to require the knowledge of higher order correlations. At any rate, the energy function (63) corresponds to a gapped spectrum since $k \rightarrow 0$ fluctuations in the quantities $t$ and $\varepsilon$ incur a finite cost. This cost, and hence the gap, are proportional to the quantities $\alpha_{i}$. Combined with Eqs. (25) and (44), and upon inclusion of square-gradient terms, Eq. (59) constitutes a field theory. We stress that the negative sign of the coefficient $a_{2}$, if any, would come about automatically in a self-consistent treatment. Once $a_{2}<0$, we arrive at a symmetry broken version of Eq. (59), such as that given in Eq. (63).

To perform a basic check on the internal consistency of this description we recall that by construction, out-ofphase motions of the ions comprising the primitive cells will cause variation in the parameters $t$ and $\varepsilon$. Denoting the amplitude of this motion with $\delta r$, one obtains, per particle:

$$
E=\frac{1}{2}\left[\alpha_{1}(\partial t / \partial r)^{2}+\alpha_{2}(\partial \varepsilon / \partial r)^{2}\right](\delta r)^{2},
$$

The quantities $(\partial t / \partial r)^{2}$ and $(\partial \varepsilon / \partial r)^{2}$ correspond with $(V / a)^{2}$ from Eqs. (34) and (36). This notion, together with Eq. (64), yields a simple qualitative expression:

$$
E=\frac{V^{2}}{2 W a^{2}}(\delta r)^{2} .
$$

At the same time, a spatially homogeneous $\delta r$ corresponds with the $k=0$ optical phonon. Equating the above expression with the kinetic energy of the vibrational motion of a bond at the Debye frequency from Eq. (49), one obtains

$$
\mu \omega_{D}^{2} \sim \frac{V^{2}}{W a^{2}} .
$$

This can be profitably rewritten by noting that $\omega_{D} a$ gives the speed of sound $c_{s}$, up to a numerical factor of order one, while $\left(\mu / a^{3}\right) c_{s}^{2}$ is the elastic constant,, 90 call it $K$, again up to a factor of order one. Thus,

$$
K a^{3} \sim \frac{V^{2}}{W} .
$$

The l.h.s. usually is of the order of a few electron volts, in actual materials $\frac{91,92}{1}$ The r.h.s. is also of the order of a few eVs, an encouraging sign. Note also that the scaling above is consistent with Eq. (38). The above equation suggests the elastic properties of a solid are related in a simple way to the (logarithmic) derivative of the on-site energies and transfer integrals with respect to the bond length, on the one hand, and the band width, on the other hand.

Besides highlighting this relation, the present field theory drives home the intrinsic connection between the fluctuations of the order parameter for the metal-insulator transition and the long-wavelength optical phonons. 
Thus the temperature-driven increase in the band gap can be thought of as caused by the increasingly large fluctuations in this order parameter.

Further insight can be obtained by adopting a specific functional form for the functional. For simplicity, we explicitly treat only the longitudinal component of the order parameter. (The transverse mode is gapped, too!) Assuming the higher order terms is a quartic:

$$
E=\frac{a_{2}}{2} \Delta^{2}+\frac{a_{4}}{4} \Delta^{4}, \quad(\Delta \geq 0),
$$

one obtains that $\Delta_{0}^{2}=a_{2} / a_{4}$ and, consequently, that

$$
\omega_{D}^{2} \propto \frac{1}{W} \propto \Delta_{0}^{2} .
$$

This relation explicitly demonstrates that the finite excitation gap for the optical phonon is directly tied to the non-zero expectation value of the order parameter. One notices at least a superficial similarity with the field theory of electroweak interactions, where the mass of the (vector) $\mathrm{W}$ and $\mathrm{Z}$ bosons is determined by the expectation value of the Higgs field. In the present context, the role of the vector bosons is played by the optical phonons while the forbidden gap $\Delta$ is the Higgs field itself. We further note that the longitudinal polarization of each of the vector boson "eats up" the Goldstone mode that would have been generated as a result of symmetry breaking; this we saw explicitly when discussing the putative picture in Fig. 5 This notion seems consistent with the general observation that when broken symmetry is not only continuous, but is also a gauge symmetry, Goldstone modes ends up being "eaten" by longitudinal components of massive fields $\stackrel{93}{ }$ We note that the dimerization does represent a lowering of a gauge symmetry since acoustic phonons are gauge particles $\underline{94}$

No such similarity is obvious for discontinuous transitions, however. In this case, the simplest form of the functional would be

$$
E=\frac{a_{2}}{2} \Delta^{2}+\frac{a_{3}}{3} \Delta^{3}+\frac{a_{4}}{4} \Delta^{4}, \quad(\Delta \geq 0)
$$

where now $a_{2}>0$ and $a_{3}<0$. Such discontinuous transitions would be the norm rather than exception in systems with harsh close-range repulsion since the third order terms is favored by closed-packed motifs $\underline{81,85,95}$ Elementary calculations show that neither the location $\Delta_{0}$ nor the curvature of the bulk term at $\Delta_{0}$ are simply related to the band width $W$. The lack of an explicit connection in this case is, perhaps, not too surprising. For instance, the insulator-to-metal transition in $\mathrm{Si}$ or Ge coincides with the melting of these solids whereby the coordination number increases from 4 to roughly $6 \underline{\underline{96}}$ The liquid-to-solid transition is particularly challenging to describe systematically because the elementary excitation above and below the transition are so different: $85,97 \mathrm{In}$ the solid, the appropriate degree of freedom is the bond vibration (the phonon) while in the liquid, it is density fluctuations.

\section{CONCLUDING REMARKS}

We have established an intrinsic connection between the presence of an insulating gap and the existence of optical phonons. The reason is that in the presence of non-closed shell bonding, electronic excitations across the gap and local polarization both involve charge transfer among atoms and inter-atomic spaces. This necessitates that the primitive cell of a crystalline solid should have at least two atoms, hence, have optical phonons. By some accounts, the distinction between acoustic and optical phonons is only formal and has to do with how one book-keeps the vibrational modes. Yet we have seen the presence of a gap in the vibrational spectrum is significant in that it signals a lowering of the symmetry.

While in the presence of optical phonons, the material could be either a metal or insulator, their absence necessarily implies the material must be a metal. A notable exception is weakly bound condensates made of closed shell atoms. In this case, excitations across the gap do not have to involve charge transfer between distinct atoms. Another arguable exception is the Wigner crystal, which nonetheless must have a lower symmetry than that of the bcc lattice, at least transiently, by exhibiting antiferromagnetism.

The existence of the optical phonons leads to a wealth of phenomena, such as the temperature dependence of the optical gap and the width of the Urbach tail of localized states below the mobility edge, in crystalline and amorphous insulators alike. These phenomena are intrinsically related and were the original motivation for the present study.

The essential role of symmetry-breaking in the emergence of the optical phonons prompted us to take a fieldtheoretical perspective on the temperature dependence of the insulating gap and its appearance in the first place. We have sketched the structure of a field theory, in which the role of the order parameter is played by the insulating gap itself. The phonon frequency scales with the expectation value of the gap, implying a common cause. (In metals exhibiting optical phonons, interactions underlying this common cause are also present, of course, but do not lead to the metal-insulator transition.) The temperature-driven narrowing of the gap simply corresponds with the temperature driven increase in the fluctuations of the order parameter.

For the present field theory, we wrote down what are essentially non-interacting Hamiltonians for the electrons and phonons. The various interactions are encapsulated in the part of the functional that governs the spatial distribution of the band gap. This part is highly non-linear and generally very complicated. In the present treatment, much of this non-linearity comes about because of the nuclear motions and the possibility of displacive instabilities. In actuality, electronic interactions should be included more explicitly since these generally affect the electronic motions even when the nuclei and insulating gap are fixed. 
Already at the level of the simple molecular orbital theory, the metal-insulator transition is seen as a breaking of continuous symmetry. Yet one can show explicitly that such breaking does not lead to the emergence of Goldstone modes. It is interesting that during the transition from delocalized to localized electronic states for random Hamiltonians of the type in Eq. (10) the broken symmetry is also continuous and that the Goldstone theorem is violated, too $\underline{98}$ In the latter description, the order parameter is the electrical conductivity. We have seen that in common materials, both a localized tail of states and a (stabilized) mobility both require the presence of optical phonons in the first place. The emergence of the optical phonons thus seems to supersede the physics of Anderson localization in the metal-to-insulator transition, except, perhaps, in specially engineered "dirty" systems. Perhaps, most common examples of such dirty systems are photonic insulators, such as milk. In any event, such random Hamiltonians are still an invaluable way to approach the question of the functional form of the tail of localized states $\stackrel{31-33}{-}$ We have speculated that the concomitant localization of the vibrational modes causes a very abrupt drop-off in the auto-correlation for the effective random potential acting on the electrons. Let us add here that it seems likely that this vibrational localization also explains why the configurational entropy of strongly interacting liquids can be often quantified by calibrating the entropy of the liquid by the entropy of weakly interacting liquids made of closed-shell molecules $\underline{\underline{54}}$

We hope that the present take on the metal-insulator transition, in conjunction with structural degrees of freedom, will be useful in the area of aperiodic charge density waves. A connection can be made with the work of Schmalian and Wolynes on the intrinsic instability of classical Coulomb systems toward vastly degenerate, aperiodic stripe phases, 99.100 a picture much richer than that anticipated by Wigner almost a century ago $\underline{85,101,102}$ The present development provides what is essentially a classical density functional theory that could be generalized to aperiodic patterns, as in Ref. 99. We observe that the order parameter must be at least two-component. Yet there are some intrinsically quantum aspects of electronic assemblies that can not be done away with even if one neglects the presence of spins, which we have largely done here except during the discussion of Wigner solids. Indeed, interfaces between distinct CDW solutions will generally host very special midgap electronic states that are topological in origin $\frac{48,49}{49}$ and are similar to the solitonic midgap states in conjugated polymers, $\stackrel{44,46}{\underline{4}}$ This important topic has not been discussed here, to be covered in a future submission.

Finally, we note that despite a superficial similarity between the creation of electron-hole pairs and electronpositron pairs, the latter does not seem to show any Urbach-like tail in the density of states: The electron/positron mass seems to be strictly bound from below by its apparent rest mass. Perhaps this fact can be interpreted, in chemical terms, as the vacuum itself being composed of "closed-shell" units whose quantum numbers all vanish, similarly to how noble gases have zero charge, orbital momentum, and spin, while requiring substantial energy to open up the shell.

Acknowledgments: We thank Peter G. Wolynes, Jon C. Golden, and Alexey Lukyanov for stimulating discussion. The financial support was graciously provided by the NSF Grant CHE-1465125 and the Welch Foundation Grant E-1765.
* vas@uh.edu

1 F. Urbach, Phys. Rev. 92, 1324 (1953).

2 W. Martienssen, J. Phys. Chem. Solids 2, 257 (1957).

3 I. Lifshitz, Adv. Phys. 13, 483 (1964).

${ }^{4}$ I. T. Godmanis, A. N. Trukhin, and K. Hübner, Phys. Stat. Solid. (b) 116, 279.

${ }^{5}$ R. A. Street, T. M. Searle, I. G. Austin, and R. S. Sussmann, J. Phys. C 7, 1582 (1974).

6 T. Arai, S. Komiya, and K. Kudo, JNC 18, 295 (1975).

7 G. D. Cody, T. Tiedje, B. Abeles, B. Brooks, and Y. Goldstein, Phys. Rev. Lett. 47, 1480 (1981).

8 G. D. Cody, in Semiconductors and Semimetals, edited by B. J. Pankove (Academic Press, New York) pp. 11-82.

9 J. R. Zakis and H. Fritzsche, Phys. Status Sol. (b) 64, 123.

10 H. Y. Fan, Phys. Rev. 78, 808 (1950).

11 H. Y. Fan, Phys. Rev. 82, 900 (1951).

12 H. Fröhlich, Proc. Roy. Soc. A 160, 230 (1937).

13 H. B. Callen, Phys. Rev. 76, 1394 (1949).

14 C. H. Grein and S. John, Phys. Rev. B 41, 7641 (1990).

15 W. M. Haynes, ed., CRC handbook of chemistry and physics (CRC press, 2011).
16 L. Cândido, B. Bernu, and D. M. Ceperley, Phys. Rev. B 70, 094413 (2004).

17 N. D. Drummond and R. J. Needs, Phys. Rev. Lett. 102, 126402 (2009).

18 K. P. ODonnell and X. Chen, Applied Physics Letters 58, 2924 (1991).

19 Y. Varshni, Physica 34, 149 (1967).

${ }^{20}$ L. Tichý and H. Tichá, Materials Letters 15, 198 (1992).

21 D. Monroe and M. A. Kastner, Phys. Rev. B 33, 8881 (1986)

22 D. Emin, Comments Solid State Phys. 11, 35 (1983).

23 D. Emin, Comments Solid State Phys. 11, 59 (1983).

${ }^{24} \mathrm{D}$ Emin and T. Holstein, Phys. Rev. Lett. 36, 323 (1976)

25 D. Emin and M.-N. Bussac, Phys. Rev. B 49, 14290 (1994)

26 Q. Zhang and P. Phillips, J. Chem. Phys. 87, 2370 (1987).

27 D. Emin, "The hall effect in hopping conduction," in The Hall Effect and Its Applications, edited by C. L. Chien and C. R. Westgate (Plenum Press, New York, 1979) p. 281.

28 N. F. Mott and E. A. Davis, Electronic Processes in Non- 
crystalline Materials (Clarendon Press, Oxford, 1979).

29 J. Tauc, "Optical properties of amorphous semiconductors," in Amorphous and Liquid Semiconductors, edited by J. Tauc (1974) pp. 159-220.

30 L. D. Landau and E. M. Lifshitz, Quantum Mechanics (Pergamon Press, 1981).

31 S. John, C. Soukoulis, M. H. Cohen, and E. N. Economou, Phys. Rev. Lett. 57, 1777 (1986)

32 S. John, M. Y. Chou, M. H. Cohen, and C. M. Soukoulis, Phys. Rev. B 37, 6963 (1988).

33 M. H. Cohen, M. Y. Chou, E. N. Economou, S. John, and C. M. Soukoulis, IBM Journal of Research and Development 32, 82 (1988).

34 P. Pyykkö, Chem. Rev. 97, 597 (1997).

35 A. Zhugayevych and V. Lubchenko, J. Chem. Phys. 133, 234503 (2010).

36 J. K. Burdett and T. J. McLarnan, J. Chem. Phys. 75, 5764 (1981).

37 P. B. Littlewood, J. Phys. C 13, 4875 (1980).

38 A. Lukyanov and V. Lubchenko, J. Chem. Phys. 147, 114505 (2017).

39 J. C. Golden, V. Ho, and V. Lubchenko, J. Chem. Phys. 146, 174502 (2017)

40 J. J. P. Stewart, "Stewart Computational Chemistry, Colorado Springs, CO, USA," HTTP://OpenMOPAC.net. Accessed August 1, 2018.

41 J. J. Stewart, J. Mol. Model 19, 1 (2013).

42 A. Lukyanov, J. C. Golden, and V. Lubchenko, J. Phys. Chem. B 122, 8082 (2018).

43 R. E. Peierls, Quantum Theory of Solids (Clarendon Press, Oxford, 1955).

44 A. J. Heeger, S. Kivelson, J. R. Schrieffer, and W. P. Su, Rev. Mod. Phys. 60, 781 (1988).

45 T. A. Albright, J. K. Burdett, and M.-H. Whangbo, Orbital Interactions in Chemistry (Wiley, Hoboken, NJ, 2013).

46 M. J. Rice and E. J. Mele,

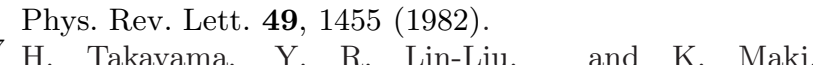
Phys. Rev. B 21, 2388 (1980).

48 A. Zhugayevych and V. Lubchenko, J. Chem. Phys. 132, 044508 (2010).

49 A. Zhugayevych and V. Lubchenko, J. Chem. Phys. 133, 234504 (2010).

50 W. Kohn, Phys. Rev. Lett. 19, 439 (1967).

51 V. Lubchenko, J. Phys. Chem. B 110, 18779 (2006).

52 Elementary Excitations in Solids (W. A. Benjamin, New York, 1963).

${ }^{53}$ L. D. Landau and E. M. Lifshitz, Statistical Mechanics (Pergamon Press, New York, 1980).

54 V. Lubchenko and P. G. Wolynes, J. Chem. Phys. 119, 9088 (2003).

55 A. A. Ovchinnikov and M. Y. Ovchinnikova, JETP 29, 688 (1969).

56 G. Grimvall and S. Sjödin, Phys. Scripta 10, 340 (1974).

57 N. W. Ashcroft and N. D. Mermin, Solid State Physics (Harcourt Brace College Publishers, Fort Worth, 1976).

58 P. Silas, J. R. Yates, and P. D. Haynes, Phys. Rev. B 78, 174101 (2008).

59 H. Katzke and P. Tolédano, Phys. Rev. B 77, 024109 (2008).

60 J. P. Attfield, Solid State Sciences 8, 861 (2006).

61 B. Monserrat, N. D. Drummond, C. J. Pickard, and R. J. Needs, Phys. Rev. Lett. 112, 055504 (2014).
62 J. K. Burdett, Chemical Bonding in Solids (Oxford University Press, 1995).

63 J. K. Burdett, Chem. Soc. Rev. 23, 299 (1994).

64 C. Brouder, G. Panati, M. Calandra, C. Mourougane, and N. Marzari, Phys. Rev. Lett. 98, 046402 (2007).

65 S. F. Boys, Rev. Mod. Phys. 32, 296 (1960).

66 C. Edmiston and K. Ruedenberg, Rev. Mod. Phys. 35, 457 (1963)

67 P. W. Anderson, Phys. Rev. 181, 25 (1969).

68 P. W. Anderson, Phys. Rev. Lett. 21, 13 (1968).

69 W. von Niessen, The Journal of Chemical Physics 56 , 4290 (1972).

70 P. G. Perkins and J. J. P. Stewart, J. Chem. Soc., Faraday Trans. II 76, 520 (1980).

71 I. N. Levine, Quantum chemistry (Prentice Hall, 2009) 6th edition, page 714 .

72 V. Labet, R. Hoffmann, and N. W. Ashcroft, The Journal of Chemical Physics 136, 074504 (2012).

73 B. Monserrat, N. D. Drummond, P. Dalladay-Simpson, R. T. Howie, P. López Ríos, E. Gregoryanz, C. J. Pickard, and R. J. Needs, Phys. Rev. Lett. 120, 255701 (2018).

74 E. Fawcett, Rev. Mod. Phys. 60, 209 (1988).

75 A. Prekul and S. Sudareva, Fizika Metallov I Metallovedenie 46, 956 (1978).

76 R. Pynn, W. Press, S. M. Shapiro, and S. A. Werner, Phys. Rev. B 13, 295 (1976).

77 J. K. Burdett and S. Lee, J. Amer. Chem. Soc. 105, 1079 (1983).

78 G. A. Papoian and R. Hoffmann, Angew. Chem. Int. Ed. 39, 2408 (2000).

79 K. Fuchs and H. H. Wills, Proc. Royal Soc. 151, 585 (1935).

80 R. A. ColdwellHorsfall and A. A. Maradudin, J. Math. Phys. 1, 395 (1960).

81 V. Lubchenko, Adv. Phys. 64, 283 (2015).

82 L. Landau, Phys. Z. Sowjet. 11, 26 (1937), English translation in "Collected Papers of Landau", 1965, Gordon and Breach.

83 L. Landau, Phys. Z. Sowjet. 11, 545 (1937), English translation in "Collected Papers of Landau", 1965, Gordon and Breach.

84 S. A. Brazovskii, JETP 41, 85 (1975).

${ }^{85}$ P. W. Anderson, Basic Notions of Condensed Matter Physics (Benjamin Cummins, Menlo Park, CA, 1984).

86 P. W. Anderson, Phys. Rev. 130, 439 (1963).

87 W. Kohn and L. J. Sham, Phys. Rev. 140, A1133 (1965).

88 W. P. Su, J. R. Schrieffer, and A. J. Heeger, Phys. Rev. B 22, 2099 (1980)

89 M. Berciu and S. John, Phys. Rev. B 69, 224515 (2004).

90 L. D. Landau and E. M. Lifshitz, Theory of Elasticity (Pergamon Press, New York, 1986).

91 V. Lubchenko and P. G. Wolynes, Phys. Rev. Lett. 87, 195901 (2001).

${ }^{92}$ V. Lubchenko and P. G. Wolynes, Adv. Chem. Phys. 136, 95 (2007), https://arxiv.org/abs/cond-mat/0506708.

93 M. E. Peskin and D. V. Schroeder, Quantum Field Theory (Addison-Wesley, 1995).

94 H. Kleinert, Phys. Lett. A 97, 51 (1983).

95 S. Alexander and J. McTague, Phys. Rev. Lett. 41, 702 (1978).

96 N. F. Mott, Conduction in Non-crystalline Materials (Clarendon Press, Oxford, 1993).

97 V. Lubchenko, Proc. Natl. Acad. Sci. U. S. A. 114, 3289 (2017). 
98 A. McKane and M. Stone, Annals of Physics 131, 36 (1981).

99 J. Schmalian and P. G. Wolynes, Phys. Rev. Lett. 85, 3456 (2001).
100 J. Schmalian and P. G. Wolynes, MRS Bull. 30, 433 (2005).

101 E. Wigner, Phys. Rev. 46, 1002 (1934).

102 E. Wigner, Trans. Faraday Soc. 34, 678 (1938). 